\title{
Collective excitations in liquid deuterium: Neutron-scattering and correlated-density-matrix results
}

\author{
F. J. Bermejo \\ Instituto de Estructura de la Materia, Consejo Superior de Investigaciones Cientificas, Serrano 123, E-28006 Madrid, Spain \\ F. J. Mompeán \\ Rutherford Appleton Laboratory, Chilton, Didcot, Oxon., OX11 OQX, United Kingdom \\ M. García-Hernández \\ Instituto de Estructura de la Materia, Consejo Superior de Investigaciones Cientificas, Serrano 123, E-28006 Madrid, Spain \\ J. L. Martínez* \\ Institut Laue Langevin, 156X, F-38042 Grenoble CEDEX, France \\ D. Martin-Marero and A. Chahid \\ Instituto de Estructura de la Materia, Consejo Superior de Investigaciones Cientificas, Serrano 123, E-28006 Madrid, Spain
}

\author{
G. Senger and M. L. Ristig
}

Institut für Theoretische Physik, Universität zu Köln, D-5000 Köln 41, Germany

(Received 2 July 1992; revised manuscript received 25 September 1992)

\begin{abstract}
The collective dynamics of liquid deuterium is studied by means of inelastic neutron scattering (INS) at $20.14 \mathrm{~K}$ and under 2 bars of applied pressure using two different incident energies. The low-incident-energy (high-energy-resolution) spectra are studied in detail by means of a time-of-flight (TOF) technique, whereas the finite-frequency response is investigated using a higher incident energy on a triple-axis spectrometer (TAS). The main finding of our work is the observation by the latter experimental technique of well-defined collective excitations up to a momentum transfer of $3.8 \AA^{-1}$. Comparisons are established with the dispersive behavior observed in other liquid systems as well as with the predictions from recent correlated-density-matrix calculations.
\end{abstract}

\section{INTRODUCTION}

The dynamical response of liquid hydrogens (mainly $\mathrm{H}_{2}$ and $\mathrm{D}_{2}$ ) at time and length scales comparable with the relevant intermolecular processes (i.e., a few picoseconds and angstroms) has attracted considerable attention from the early days of neutron scattering. ${ }^{1}$ Although a number of studies have been reported on the low-energy dynamics (i.e., quasielastic scattering) of liquid and dense-gas phases, ${ }^{1,2}$ as well as on the high-momentum-transfer (i.e., deep inelastic) region in the solid and liquid phases of $\mathrm{H}_{2},{ }^{3}$ rather few attempts have been registered where the dispersion curves for solid ${ }^{4}$ or liquid ${ }^{5,6}$ samples have been measured.

This situation, however, contrasts with the need to provide dispersion data for these liquids where, due to their low viscosity, collective excitations are expected to be well-defined entities for a vast range of momentumtransfer values within the kinematical range accessible by neutron spectroscopy. An additional source of interest is the search for manifestations of some quantum phenomena in the collective dynamical behavior of these molecular liquids where noticeable quantum effects are expected at temperatures close to the triple point (the thermal wavelength, $\lambda_{T}$, for $\mathrm{D}_{2}$ in the liquid range near this point is about $1.3 \AA$ ). These systems constitute an intermediate case between ${ }^{4} \mathrm{He}$ where the thermal wavelength at $T=2.1 \mathrm{~K}$ is about $4.6 \AA$, and Ne where $\lambda_{T}=0.6$ $\AA$ at $T=27 \mathrm{~K}$. It is worth mentioning that the interest in the collective dynamical properties of the liquid hydrogens has been reawakened by the expectations raised from some calculations regarding the possibility of supercooling liquid para-hydrogen ${ }^{7}$ as well as from the prediction of the existence of a superfluid phase in clusters of $\left(\right.$ para- $\left.\mathrm{H}_{2}\right){ }^{8}$

The purpose of this paper is, therefore, to provide new and up-to-date information regarding the collective dynamics of liquid normal deuterium in the bulk phase at temperatures just above melting. In order to achieve our purpose we have performed neutron inelastic scattering experiments with two different incident energies. The values of these energies $(4.8 \mathrm{meV}$ and $34.9 \mathrm{meV})$ have been chosen, with due regard to the estimate of the isothermal sound velocity, respectively, below and above the kinematical excitation threshold for soundlike excitations (ca.7 meV for wave vectors of $1 \AA^{-1}$ ).

The reason to choose deuterium instead of para- $\mathrm{H}_{2}$ was motivated by the fact that, in the latter case, due to 
the conversion to incoherently scattering ortho-hydrogen (ortho- $\mathrm{H}_{2}$ ), the incident neutron energy has to be lower than the one associated to the ortho-para conversion (14.5 meV), a fact which precludes the measurement of the dispersion curve at momentum-transfers below 1 $\AA^{-1}$, due to kinematical restrictions. On the contrary, for an equilibrium mixture of $\mathrm{D}_{2}$ with a para- $\mathrm{D}_{2}$ concentration of $1 / 3$, the cross section for thermal or cold neutrons is mostly coherent even if the incident neutron energy surpasses the ortho- to para- $\mathrm{D}_{2}$ conversion energy (about $7.4 \mathrm{meV}$ ).

We also include a study of the high-energy-resolution (low-incident energy) spectra of the polycrystalline (hcp) solid at $T=10 \mathrm{~K}$ in order to provide a comparison with the liquid, since the only data found in the literature corresponds to rather old measurements, ${ }^{1}$ performed using, both energy resolution and counting statistics far below the capabilities of the present-day spectrometers. On the other hand, for the high-energy dynamics of the solid phase we rely on the dispersion data for single crystals of ortho- $\mathrm{D}_{2}$ measured using a TAS instrument by Nielsen. ${ }^{4}$

A preliminary account of the results concerning the TAS data has already been given, ${ }^{6}$ and the present work provides a complete comparison between data measured using different excitation energies, a fact which enables the separation of the low-energy contributions (singleparticle quantities) from the total coherent response.

The sketch of the paper is as follows; Sec. II will give some details about the experimental techniques; the data analysis procedures and the employed approximations are described next in Sec. III. The most relevant details of the obtained results are then given in Sec. IV where a comparison is established with the predictions of the correlated-density-matrix variational approach. Finally the discussion and main conclusions are given in Sec. V.

\section{EXPERIMENTAL DETAILS}

Two sets of experiments were carried out using different incident energies and energy resolutions.

\section{A. Low incident energy}

High-resolution measurements were carried out using the IN6 time-of-flight (TOF) spectrometer located on one of the cold neutron guides at the Institut Laue Langevin (ILL), Grenoble (France). The neutron scattering double differential cross sections were measured using an incident wavelength of $4.12 \AA$ (corresponding to an incident energy $E_{i}=4.82 \mathrm{meV}$ and incident wave vector $\left.k_{i}=1.53 \AA^{-1}\right)$. The sample was prepared from condensation of gaseous deuterium (99.99\%) into a cylindrical cell of dimensions $10 \times 50 \mathrm{~mm}$, which contained five cadmium spacers (with thickness $0.3 \mathrm{~mm}$ ) inserted in order to reduce the contribution of multiply scattered neutrons. The sample was condensed in situ into a standard orange cryostat and the sample temperature was measured with a platinum resistance directly attached to the cylindrical cell. The measurements were carried out at $10 \mathrm{~K}$, in the solid hexagonal close packed phase and $20.14 \mathrm{~K}$ in the liquid under an applied pressure of 2 bars. From the ratio of the integrated areas of the quasielastic and inelastic rotational peaks in the solid phase, the concentration of the para-species was found to be approximately $32 \%$. Calibration runs with the empty cell as well as with vanadium foil rolled around the container were also performed in order to normalize the measured intensities. The crystal formation and melting were monitored from plots of the wave-vector dependence of the elastic intensity $S(Q, E=0)$.

The cross-section data were converted into constantangle dynamic structure factors $S(\Theta, E)$ using the INX (Ref. 9) suite of programs. The contribution of multiply scattered neutrons was evaluated for one of the volume elements between cadmium spacers using the DISCUS code. ${ }^{10}$ The integrated contribution of multiple scattering was found to be less than $5 \%$ of the total intensity. As an example of the relative importance of this contribution, the ratio of single to total scattering events for $Q=1 \AA^{-1}$ amounts to 0.956 at the elastic, 0.99 at $1 \mathrm{THz}$ $(0.926$ at $-1 \mathrm{THz})$ and 0.999 at $2 \mathrm{THz}(0.887$ at $-2 \mathrm{THz})$.

The constant-angle spectra were finally converted into $S(Q, E)$ spectra by means of the INGRID code. ${ }^{11}$

The achieved energy resolution was about $35 \mu \mathrm{eV}$, estimated from the vanadium runs as the half-width at half-height (HWHM) of the elastic peak.

\section{B. High-incident energy}

The high-incident-energy experiment was carried out using the IN8 triple-axis (TAS) spectrometer located on one of the thermal beam tubes of the ILL. The same container was used and the incident wavelength was set to $1.53 \AA\left(E_{i}=34.95 \mathrm{meV}, k_{i}=4.1 \AA^{-1}\right)$. A rather restrictive collimation was used in order to achieve an acceptable dynamic range free from the contamination from the direct beam. Most of the experiments were done using the above-quoted incident wave vector which gives an energy resolution measured with a vanadium standard of $2.8 \mathrm{meV}$. Some experiments were also performed using a lower incident energy (17 meV) which gave an energy resolution of $1.45 \mathrm{meV}$ (HWHM). All the spectra were measured in the constant- $Q$ and constant incident-wavevector configuration. A cylindrical vacuum tank of 1-mdiameter placed surrounding the cryostat tail and narrow collimation around the sample were used in order to reduce the contribution of diffuse scattering from the air. An absorption and container subtraction correction was applied using a generalization of the Paalman-Pings procedure to inelastic scattering. ${ }^{12}$ Multiple scattering corrections were also performed and the resolution in energy and momentum transfers were finally computed for each measured point using a modified version of the RESCAL ${ }^{13}$ code. As an indication, the achieved resolution in energy and momentum transfers were, for the elastic peak at $Q=1.0 \AA^{-1}$, of about $2.5 \mathrm{meV}$ and $0.12 \AA^{-1}$.

All the measurements using the TAS spectrometer were carried out at $20.14 \mathrm{~K}$ and 2 bars of applied pressure. The complete melting of the sample was monitored by measurements of the elastic structure factor $S(Q, E=0)$. Its value was also used in order to normalize the measured intensities to an absolute scale. 


\section{DATA ANALYSIS}

The scattering intensity can be written as a sum of contributions from single and multiple excitations convolved with the instrumental resolution, $R(Q, E)$, as

$$
\begin{aligned}
I(Q, E)= & A\left[S^{1}(Q, E)+S^{M}(Q, E)\right] \otimes R(Q, E) \\
S^{1}(Q, E)= & S_{\text {self }}(Q, E)+S_{\text {coh.que. }}(Q, E) \\
& +S_{\text {exc }}(Q, E)
\end{aligned}
$$

where the term $S^{1}(Q, E)$ contains information about single-excitations of both diffusive ("self"), coherent quasielastic ("coh.que."), and propagative ("exc") nature, and $A$ is a global scaling constant.

At temperatures where the collective excitations are not appreciably populated, and working with neutron incident energies well below those corresponding to the lowest-lying (acoustic) modes, it is possible to observe exclusively those contributions to the intensity arising from the low-frequency dynamics (stochastic motions with an average zero frequency). Following such an approximation we shall examine first the low-energy dynamics. This becomes necessary for a proper account of the wings of the quasielastic intensity in higher energy-transfer experiments, while it helps to establish the relative importance of the different contributions shown above.

\section{A. Low-energy dynamics}

From previous work on the low-energy spectra of solid and liquid hydrogens, ${ }^{1}$ it is a well-known fact that the spectra are dominated, in the case of the solid, by an elastic peak which corresponds to $J=0 \rightarrow 0$ and $J=1 \rightarrow 1$ rotational transitions ( $J$ is the total molecular angular momentum quantum number) and a progression of inelastic peaks corresponding (in the neutron energy-gain side, $E<0$, of the spectra) to rotational transitions $J=1 \rightarrow 0$ as well as those which involve higher rotational excited states, although at the temperatures corresponding to these experiments only the $J=1$ and $J=0$ levels are thermally populated. In the liquid, additional broadening of the quasielastic line is expected to occur due to diffusive (translational) motions. In both condensed phases a small contribution to the energy shifts and intrinsic widths of the rotational lines is expected to be noticeable due to recoiling processes.

In the case of deuterium, the $J=0$ (ortho) rotational state is associated with even total nuclear spin states (with quantum number $I=0$ or 2 ), and the $J=1$ (para) state with an odd $(I=1)$ counterpart. The equilibrium concentration of para-molecules at room temperature is $1 / 3$ and a slow (expected rate ca. $0.060 \%$ per hour) conversion into the ortho-species occurs at low temperatures. The quasielastic intensity in the solid arises from $\Delta J=0$ rotational transitions and coherent elastic effects. For the purpose of establishing a comparison with the liquid, the corresponding broadening can be loosely described as caused by a "hindering" to free rotation. This hindering effect would yield a broadening of $2.7 \mu \mathrm{eV} Q^{2}$ as was predicted by Elliot and Hartmann. ${ }^{1}$

From inspection of the fully corrected, constant- $Q$ spectra for which some sample plots are shown in Fig. 1 , the following conclusions may be drawn.

(i) No broadening of the elastic peak could be detected using the achieved energy resolution in the solid. A wellresolved peak is found centered at about $-7.2 \mathrm{meV}$ which corresponds to the para-ortho rotational transition between energy levels given by $E_{\text {rot }}=B_{0} J(J+1)$ with the rotational constant for the vibrational ground state, $B_{0}=3.6 \mathrm{meV}$. The position and width of this rotational component show a weak dependence on the momentum transfer.

(ii) A strong variation in the position of energy transfers and linewidths of the rotational peak is seen upon melting. Furthermore, a noticeable broadening of the elastic peak is apparent in the liquid, showing a phase relationship with the $S(Q)$ at large momentum transfers.

(iii) Apart from the rotational peak, no signature of finite-energy response is apparent from inspection of the energy-gain side of the spectra. The absence of such a response at low-incident energies has also been confirmed by some measurements performed on the TAS spectrometer using an incident wave vector of $2.66 \AA^{-1}$.

A simplified approach was first considered in order to analyze the spectral shapes. The spectral shape for the solid (shown in Fig. 1) could be reproduced by

$$
\begin{aligned}
I_{\text {solid }}(Q, E)=A \exp (-2 W) & {[\delta(Q, E=0)} \\
& \left.+S_{\text {rot }}(Q, E)\right] \otimes R(Q, E),
\end{aligned}
$$

where the symbol $\otimes$ means convolution and the rotational contribution is given by

$$
S_{\mathrm{rot}}(Q, E)=\frac{1}{\left(2 \pi \sigma_{s}^{2}\right)^{1 / 2}} \exp \left(-\frac{\left(E-E_{p}\right)^{2}}{2 \sigma_{s}^{2}}\right),
$$

here the peak center $E_{p}$ is assumed to be a sum of a $Q$-independent part $E_{\text {rot }}$ corresponding to the rotational $J=1 \rightarrow 0$ transition and a recoil term given by $E_{r}=$ $\hbar^{2} Q^{2} / 2 M_{\text {eff }}$, the quantity $M_{\text {eff }}$ being an effective mass for the recoiling particle. The symbol $A$ stands for a scaling constant, the exponential is a Debye-Waller term, the delta component accounts for the elastic intensity and $R(Q, E)$ is the resolution function measured with the vanadium standard. The width of the rotational peak has been used as an adjustable parameter which should contain contributions arising from the broadening of the single-molecule transitions due to hindering by mutual interaction as well as a small contribution from recoil and coherent effects.

Although the approximation given above cannot be considered to be strictly valid, the fits to the experimental intensities show that the line shape is well described using this simplified treatment up to a good approximation. A comparison between the observed and fitted spectra is shown in Fig. 2.

On the other hand, due to the substantial broadening clearly apparent in the liquid, a more detailed formulation is required to account for the mass-diffusion effects present in the fluid phase. The adopted model is a sum of one and multiphonon processes which is convolved with the experimental resolution 
$I_{\text {liquid }}(Q, E)=B\left[S^{1}(Q, E)+S^{M}(Q, E)\right] \otimes R(Q, E)$,

where $B$ is a scaling constant, $S^{M}(Q, E)$ represents the multiphonon background which has been evaluated from formulas given in Ref. 14 in terms of the total density of states (DOS). ${ }^{15}$ As a model for the low-energy dynamics we have adopted

$$
\begin{aligned}
S^{1}(Q, E)= & S_{\text {self }}(Q, E) \\
& +[n(E)+1] a^{2}(Q) S(Q) \frac{E \Gamma(Q)}{E^{2}+[\Gamma(Q)]^{2}},
\end{aligned}
$$

where the first term represents the scattering from single molecules and accounts for rotational transitions as well as for recoiling processes and diffusive motions:

$$
S_{\text {self }}(Q, E)=S_{\text {free }}(Q, E) \otimes S_{\text {diff }}(Q, E) .
$$

The term $S_{\text {free }}(Q, E)$ accounts for processes involving free recoil and the relevant formulas for these contributions have been given in Eqs. (2a) and (2b) of Ref. 6. The diffusive motions giving rise to incoherent quasielastic broadening are accounted for in the contribution $S_{\text {diff }}(Q, E)$ for which a Lorentzian shape has been assumed. The peak shapes associated with this contribution therefore are modeled in terms of Voigt functions (i.e., a convolution of a Lorentzian and a Gaussian). ${ }^{16}$ The term $n(E)$ is the Bose factor, and the low-energy coherent interference effects are represented by a Lorentzian with a $Q$-dependent width $\Gamma(Q)$ and an amplitude given by a coherent molecular form factor

$$
a(Q)=2 b_{\mathrm{coh}} j_{0}\left(\frac{1}{2} Q r_{\mathrm{eq}}\right),
$$

where $b_{\text {coh }}$ is the coherent scattering length, the function $j_{0}\left(Q r_{\text {eq }} / 2\right)$ is the spherical Bessel function of zero order. Its argument is given in terms of the momentumtransfer $Q$ as well as the equilibrium internuclear separation. $S(Q)$ is the static structure factor. It should be noted that the detailed balance condition for the selfterm is explicitly included in the formulas referred to above.

The model adopted for the single-molecule scattering is of an approximate nature because of the underlying assumption of the presence of only weak anisotropic intermolecular forces. Although some more detailed treatments have appeared such as the one due to Elliot and Hartmann, ${ }^{1}$ where the effect of rotational hindering due to anisotropic interactions is taken into account, they are only applicable to the case of purely incoherent scat-
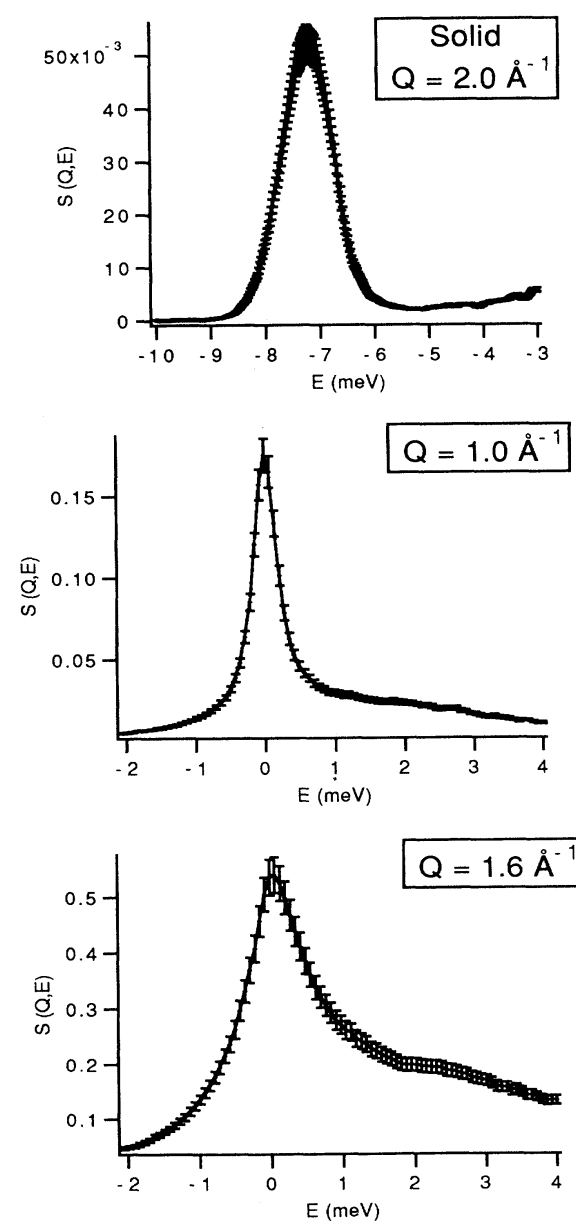
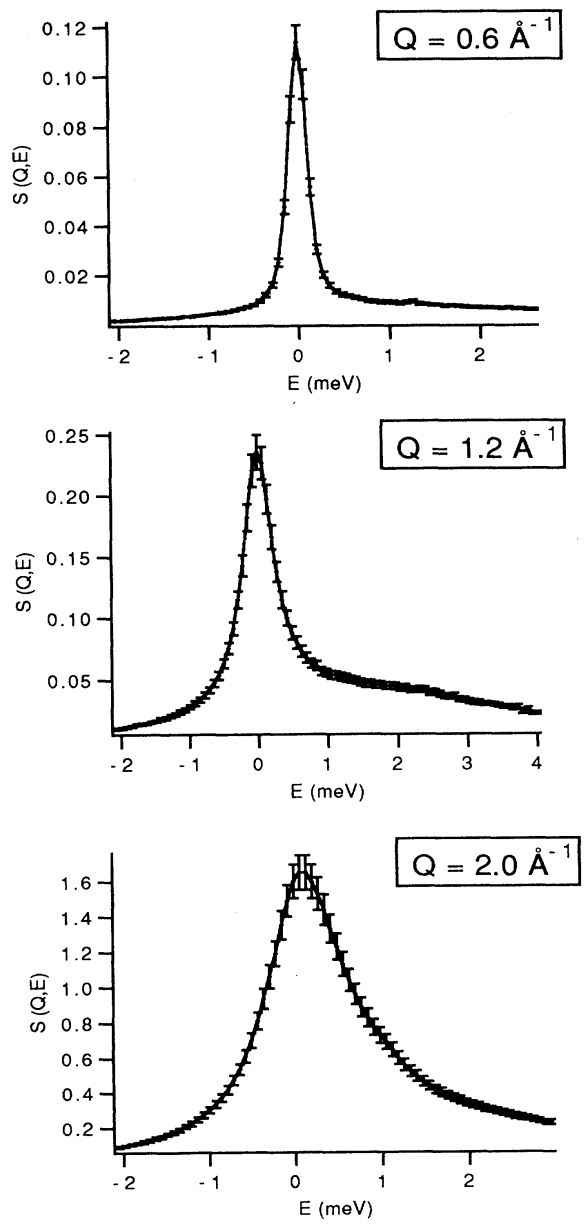

FIG. 1. Constant- $Q S(Q, E)$ dynamic structure factors for solid and liquid phases of normal deuterium as measured using the TOF spectrometer. Only the rotational $J=1 \rightarrow$ 0 is shown for the solid since no broadening of the quasielastic peak was apparent. Note the presence at $Q=1.6 \AA^{-1}$ of a broad inelastic feature centered at about $2.4 \mathrm{meV}$ and the different energy scale for the solid phase. 
tering and do not take into account the broadening due to translational diffusive motions. As an alternative, we have modeled the single-molecule contribution in terms of a freely diffusing particle with an effective mass $M_{\text {eff }}$ following the same lines of reasoning as Powles and Rickayzen. ${ }^{17}$ The wave-vector dependence of the incoher- ent broadening other than the one due to recoil motions is therefore accounted for by the diffusive contribution.

A comparison of the experimental and fitted spectra is also given in Fig. 2.

The density $Z(E)$ of states (DOS) for the polycrystal has been obtained from ${ }^{18}$

$$
I(Q, E)=\exp (-2 W)\left(\hbar Q^{2} / 2 M_{\mathrm{eff}}\right)[n(E)+1] \Delta E \frac{Z(E)}{E} \frac{1}{2} \int_{-1}^{1} S\left(Q+q_{E} z\right) d z
$$

where $\Delta E$ is the energy window employed in the present experiment, $q_{E}$ is an average wave vector over all those modes entering such energies, and the rest of the symbols retain the meaning given above. A different approach was followed for the liquid, where in order to get the correct intercept at zero frequency the procedure due to Carneiro $^{19}$ was followed. In this treatment, in order to regain the limit at $E \rightarrow 0$, the experimental intensities were divided by $E^{2} /\left[E^{2}+\left(D_{T} Q^{2}\right)^{2}\right]$, where $D_{T}$ stands for the macroscopic diffusion coefficient. Estimates for this quantity were obtained from the analysis of the quasielastic spectra as it will be described below. The resulting function $Z(E)$ is displayed in Fig. 3(b).

\section{B. Collective dynamics}

In order to analyze the TAS spectra of the nonzero frequency excitations that are clearly visible in Fig. 4, the scattering law for $S_{\text {exc }}(Q, E)$ needs to be specified. Such a contribution has been modeled in terms of a damped harmonic oscillator,

$$
S_{\text {exc }}(Q, E)=2 \pi H(Q)[1+n(E)] E D\left(Q, E^{2}\right)
$$

to take account of the effects of these elementary excitations. They are characterized by their renormalized frequency $\Omega_{Q}$ and an associated damping amplitude $\Gamma_{Q}$ and described by the function

$$
D\left(Q, E^{2}\right)=\frac{2}{\pi} \frac{\Gamma_{Q} \sqrt{\left(\hbar \Omega_{Q}\right)^{2}-\Gamma_{Q}^{2}}}{\left[E^{2}-\left(\hbar \Omega_{Q}\right)^{2}\right]^{2}+4 E^{2} \Gamma_{Q}^{2}}
$$

suitably normalized to unity, $\int_{-\infty}^{+\infty} D(Q, x) d x=1$. Function $H(Q)$ acts as an appropriate strength factor independent of the energy $E$ and may be adopted by fitting the experimental data. The semiphenomenological ansatz (9) is based on the picture of a damped harmonic oscillator. In the limit of no damping, function (9) represents a Dirac function, $\delta\left[E^{2}-\left(\hbar \Omega_{Q}\right)^{2}\right]$, with sharply peaked excitation energies $\hbar \Omega_{Q}$ and the strength factor $H(Q)$ may be related to the static structure function $S(Q)$.

The relative importance of the components (4) and (8) constituting the spectral function of the total intensity can be gauged by comparing the measured and fitted intensities shown in Fig. 5. To reduce the number of free parameters our results on the function $S_{\text {self }}(Q, E)$ and the coherent quasielastic contribution, Eq. (4), derived from the high-energy-resolution experiment are used as constants for analyzing the TAS data. In this way the number of adjustable parameters reduces to four, i.e., $\Omega_{Q}, \Gamma_{Q}, H(Q)$, and a global scaling constant $B$.

\section{RESULTS}

\section{A. Low-energy dynamics}

\section{Polycrystalline deuterium}

The inspection of the $S(\Theta, E)$ surface revealed the presence of a series of intense elastic peaks (the fundamental powder peaks) at $Q=1.99,2.12$, and $2.26 \AA^{-1}$ and a well-defined inelastic peak due to the para to ortho conversion.

The energy of the rotational peak, its linewidth, and amplitude have a significant $Q$ dependence. Such a finding is in contrast with the theoretical predictions and measurements ${ }^{1}$ carried out more than two decades ago, where no significant $Q$ dependence was found. The higher energy resolution and better counting statistics of the IN6 spectrometer have therefore provided a test of these predictions.

We have used the model proposed in Eqs. (1) and (2) to study this dependence and extract the relevant parameters.

The extrapolation $Q \rightarrow 0$ of the wave vector dependence of the linewidth gives a value of $0.45 \mathrm{meV}$ which coincides with the Elliot and Hartmann prediction but contrasts with the value of $0.76 \mathrm{meV}$ reported by Egelstaff et al. ${ }^{1}$ Also, the width of the elastic line arising from contributions from $J=1 \rightarrow 1$ and $J=0 \rightarrow 0$ transitions has to be smaller than the experimentally achieved resolution of $35 \mu \mathrm{eV}$.

A relatively small value of $7.20 \mathrm{meV}$ for the $J=1 \rightarrow 0$ transition energy was found rising up to $7.5 \mathrm{meV}$ upon melting, which is closer to the free rotor value. A possible reason for such a reduction in energy may be the coupling of rotation to lattice modes ${ }^{20,21}$ since estimates of the reduction in energy of $0.17 \mathrm{meV}$ have been reported due to this effect. A similar phenomenon has been reported for solid hydrogen ${ }^{22}$ where a dependence on the concentration of ortho- $\mathrm{H}_{2}$ has been found.

The $Q$ dependence of the amplitude of the rotational peak provides an estimate of the value of the DebyeWaller factor. Contrary to the usual need of estimating this magnitude from the intensity of the elastic peak, this transition enables the estimation of the mean-squared amplitude of vibration $\left\langle u^{2}\right\rangle$ almost free from systematic 
(a)
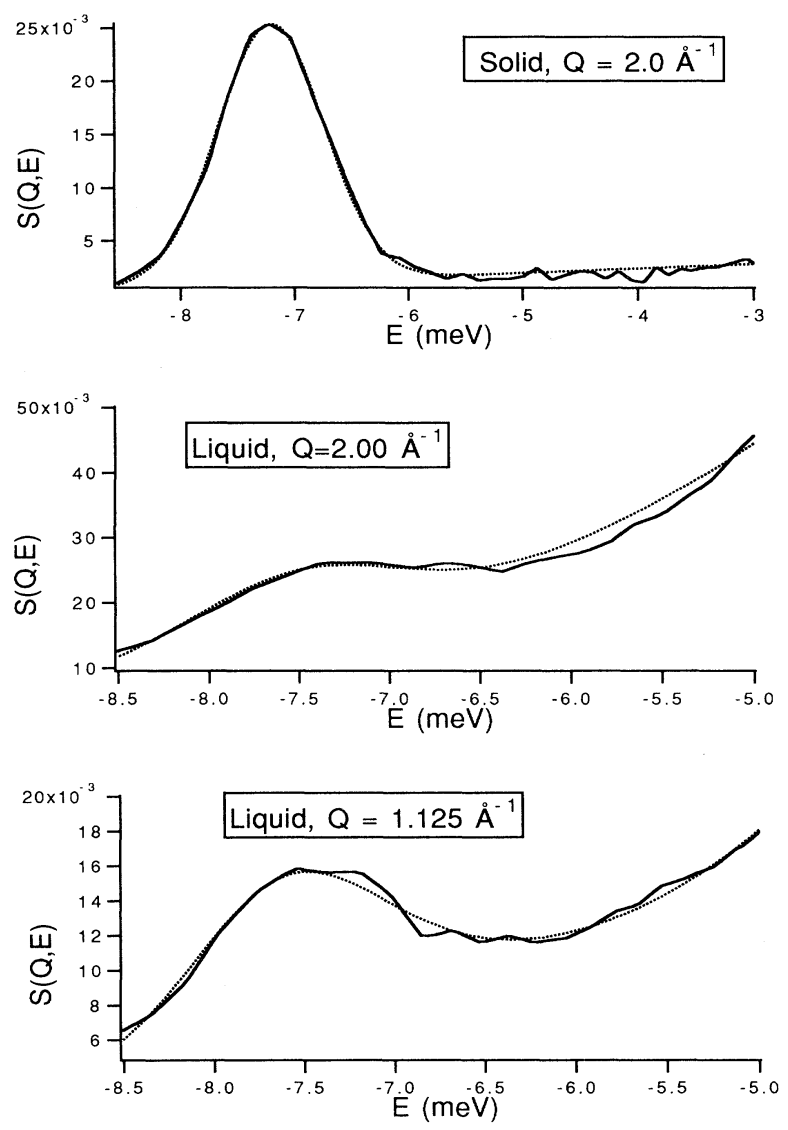

(b)
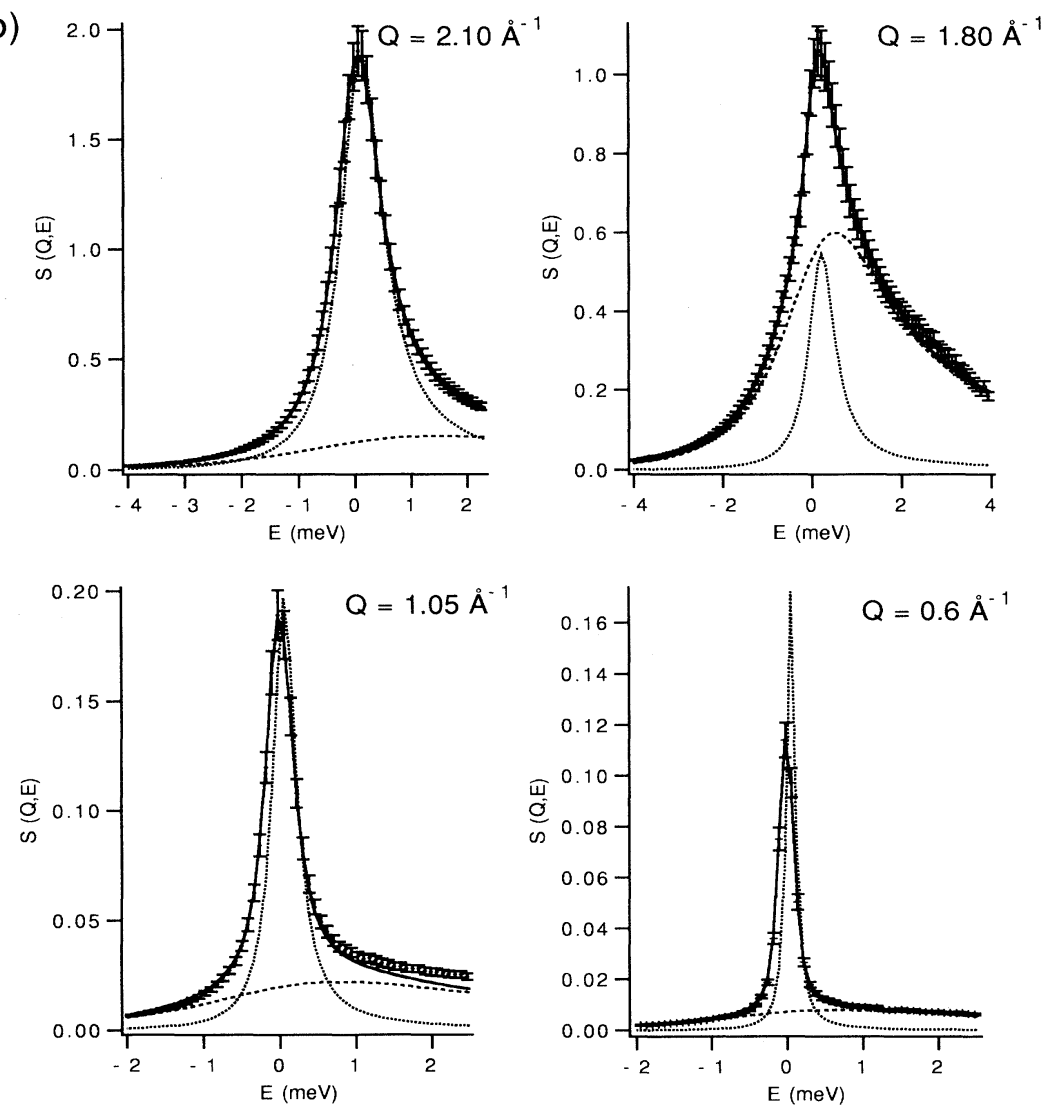

FIG. 2. Comparison of fitted and observed TOF spectra for the solid at $T=10$ $\mathrm{K}$ and the liquid at $T=20.14 \mathrm{~K}$. (a) Rotational band contours: the experimental data are shown as a solid line and the fitted model as dots. (b) Quasielastic spectra: the experimental data are shown as markers with their respective error bars and the fitted model is shown by the solid line. The incoherent contribution, $S_{\text {self }}(Q, E)$, is shown as a dotted line, and the coherent quasielastic intensity is represented by dashes. 
errors associated with small variations of the elastic intensity due to the correction procedures. ${ }^{4}$ Our analysis neglects crystal-field effects ${ }^{20}$ so that the wave-vector dependence of the intensity of this peak can be analyzed in terms of the free rotor function

$$
I_{J=1 \rightarrow 0}(Q)=A \exp \left(-\frac{1}{3} Q^{2}\left\langle u^{2}\right\rangle\right)\left[j_{1}\left(Q r_{\mathrm{eq}} / 2\right)\right]^{2},
$$

where $A$ is a scaling constant, which encompasses a normalizing factor as well as the ratio of ortho/para species, and $j_{1}\left(Q r_{\mathrm{eq}} / 2\right)$ is a spherical Bessel function with an argument given in terms of the wave vector and the equilibrium internuclear distance. The fitted function is shown in Fig. 6. The estimate for the mean-squared amplitude of vibration yields a value of $0.30 \AA^{2}$, which compares favorably with the estimate of $0.48 \AA^{2}$ previously reported
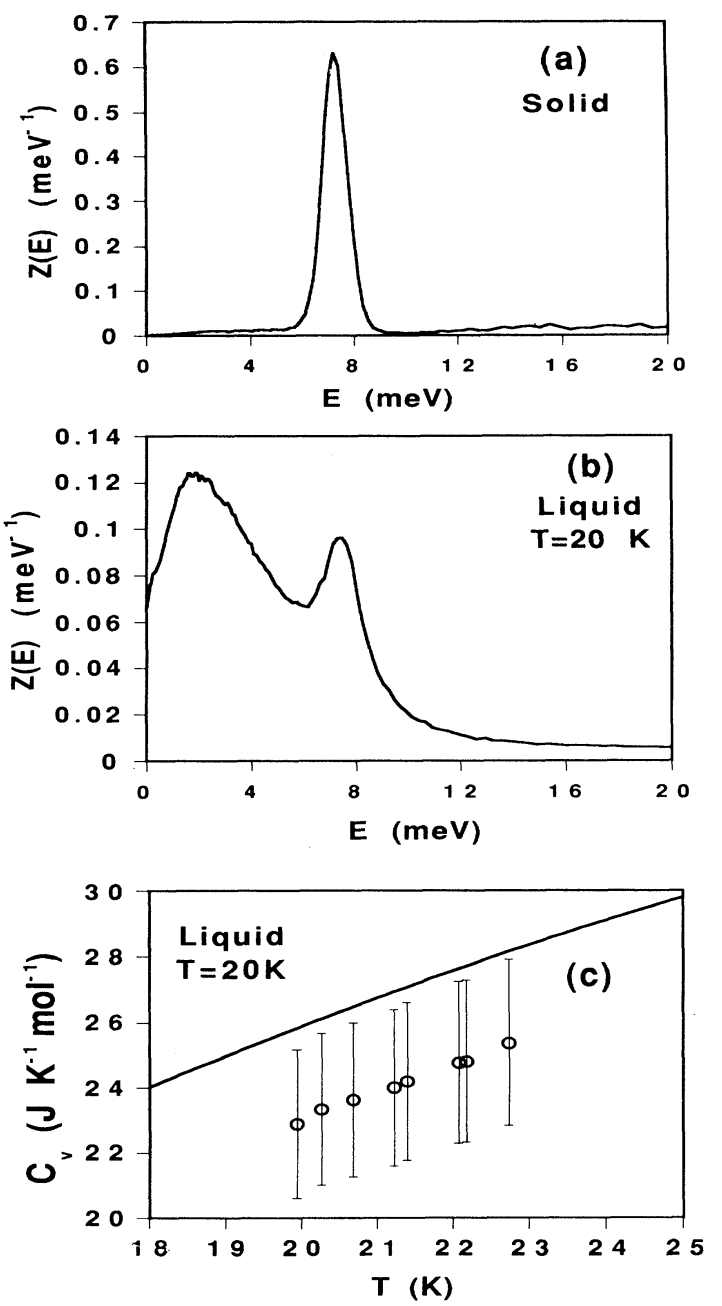

FIG. 3. The upper and middle frames show, respectively, the densities of vibrational states for the solid and liquid phases of deuterium, as obtained from the TOF data. Both curves are normalized to unit area. The bottom frame shows the calculated specific heat at constant volume for the liquid phase (solid lines). The open circles show the calorimetric data and the error bars correspond to about $10 \%$ of the measured values. for solid $\mathrm{H}_{2}$ at $T=5.4 \mathrm{~K}$ by Nielsen ${ }^{4}$ and of $0.46 \AA^{2}$ reported by Bickerman et al. ${ }^{22}$ once the different molecular masses are taken into account.

From the density $Z(E)$ of vibrational states shown in Fig. 3(a) and obtained using Eq. (7) it can be clearly seen that the only prominent feature derives from the rotational transition. The sound-mode contributions that should appear at energies of about $6 \mathrm{meV},{ }^{4}$ according to an estimate using a Debye model and the reported velocity of sound, as well as optical data, ${ }^{23}$ are too weak to be observed using this incident energy $\left(E_{0}=4.8 \mathrm{meV}\right)$. Thus, further support is given to the decomposition of spectral contributions proposed at the beginning of Sec. III.

\section{Liquid deuterium}

The most representative part of the $S(\Theta, E)$ surface is shown in Fig. 7, where two different regions are clearly seen. A relatively narrow incoherent quasielastic peak dominates the scattering pattern at low angles (up to an elastic- $Q$ value of $Q_{e}=1.5 \AA^{-1}$ ), and a strong coherent quasielastic response builds up from this value of momentum transfer showing its maximum at about $Q_{e}=2.2$ $\AA^{-1}$.

The wave-vector dependence of these peak positions and linewidths of the rotational peak in the liquid phase is shown in Fig. 8. In contrast to the solid, the peak, apart from being broader due to the presence of diffusive motions, overlaps substantially with the coherent quasielastic intensity. This fact introduces additional difficulties when trying to estimate accurately the peak position and its intrinsic width. As it can be seen from Fig. $2(\mathrm{a})$, this spectral contribution only shows a well-defined shape within a narrow range of momentum-transfer values (between 1.0 and $1.25 \AA^{-1}$ ), so that the estimated parameters are affected by relatively large errors in comparison with the solid. The rotational transition energy estimated from the extrapolation $Q \rightarrow 0$ of the peak maxima is now $7.56(12) \mathrm{meV}$, in reasonably good agreement with the values estimated from other spectroscopic ${ }^{3}$ or theoretical $^{21}$ studies.

The $Q$ dependence of the amplitude and width of the coherent and incoherent quasielastic peaks is shown in Fig. 9. Coherent effects dominate the pattern so that the maximum of the amplitude coincides with the maximum of the static structure factor $S(Q)$ at $Q \simeq 2.1 \AA^{-1}$ measured for the liquid and reported in Ref. 24. It is worth noting that the contribution from purely incoherent scattering associated with the broadening of $J=0 \rightarrow 0$ and $J=1 \rightarrow 1$ transitions that dominates the spectra at $Q$ values below $1.5 \AA^{-1}$, is rather small in the momentumtransfer range where the energy window is large enough to enable a proper analysis of the quasielastic line, since, in this range of wave vectors, such a term only amounts to a smoothly decaying background with an intensity of about $5 \%$ of the total. In contrast, an estimation based upon the static approximation ${ }^{14}$ would predict a contribution of these effects of about $26 \%$.

From the wave-vector dependence of the width of the 
spectral component $S_{\text {self }}(Q, E)$ at $Q$ values well below the maximum of the static structure factor, some estimate of the diffusion coefficient can be extracted. For this liquid the rotational motion due to its quantized nature can be safely separated from the mass-diffusion motions (i.e., the contribution of the rotational bands to the quasielastic part of the spectrum is rather weak). Consequently, the diffusive component may be analyzed within the framework of the mode-coupling theory that has been successfully applied to studies of monatomic liquids and dense gases. The $Q$ dependence of the linewidth $\Delta E(Q)$, amplitude $S_{\text {diff }}(Q, 0)$, and their product can therefore be properly analyzed in terms of the mode-coupling equations ${ }^{25}$

$\Delta E(Q)=Q^{2}\left[D_{T}-D_{T} H(\delta) Q / Q^{*}+O\left(Q^{3 / 2}\right)\right]$,

$S_{\mathrm{diff}}(Q, 0)=\frac{A}{\pi Q^{2}}\left[D_{T}^{-1}+\frac{D_{T}^{-1} G\left(\delta^{-1}\right) Q}{Q^{*}}+O\left(Q^{3 / 2}\right)\right]$,
$S_{\mathrm{diff}}(Q, 0) \Delta E(Q)=\frac{A\left[1+B(\delta) Q / Q^{*}+O\left(Q^{3 / 2}\right)\right]}{\pi}$.

Here, $D_{T}$ is the translational diffusion coefficient, $\delta=$ $D_{T} /\left(D_{T}+\nu\right), \nu$ is the kinematical viscosity, and $Q^{*}=$ $16 \pi \beta M n D_{T}^{2}$ is the wave number associated with a particle of mass $M$ immersed in a fluid with number density $n$ and at a temperature $T=1 /\left(k_{B} \beta\right)$. The functions $G\left(\delta^{-1}\right), H(\delta)$, and $B(\delta)$ were calculated employing expressions given in Ref. 26. The resulting values for the self-diffusion $D_{T}$ coefficient and the kinematical viscosity $\nu$ are $D_{T}=0.3(1)$ and 19.6(1) $\AA^{2} \mathrm{ps}^{-1}$, respectively, and the curves corresponding to these values are shown in Fig. 9.

On the other hand, in order to perform a consistency check for the obtained value of the diffusion coefficient, the $Q$ dependence of the amplitude of the elastic peak up to $Q=1.5 \AA^{-1}$ (i.e., the region where the energy win-
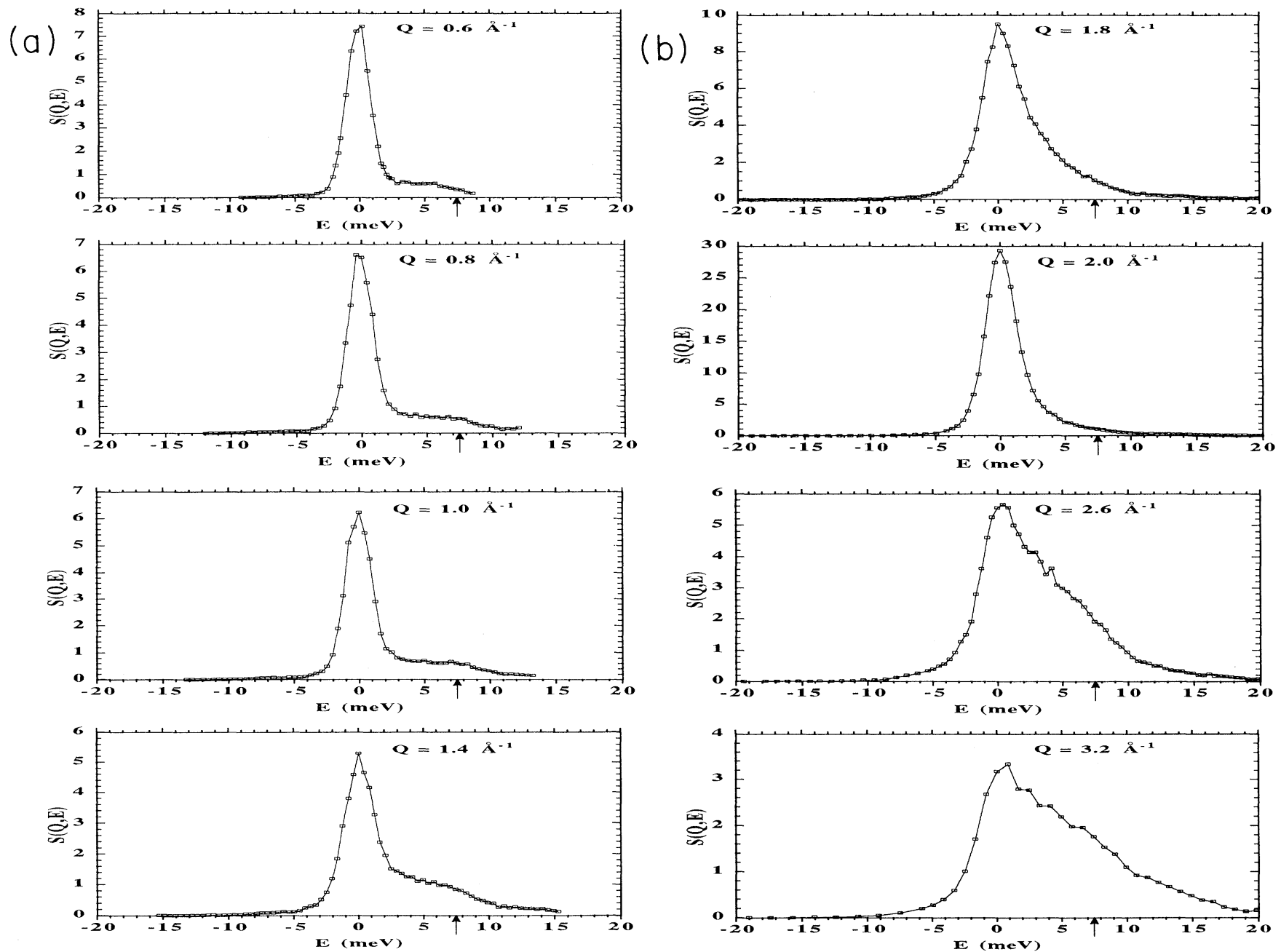

FIG. 4. A sample of the fully corrected higher energy TAS spectra for the liquid at $T=20.14 \mathrm{~K}$ at several values of the momentum transfer. Note the difference in energy-transfer scales due to kinematical restrictions. The arrows mark the position of the rotational transitions. 
dow in the constant- $Q$ spectra is too small to allow any detailed analysis of the shape, and the measured intensities are dominated by the incoherent contribution), is further analyzed in terms of an equation that includes the hindering effects studied by Elliot and Hartmann, ${ }^{1}$ as well as the diffusive component described within the mode-coupling formalism,

$$
\begin{aligned}
I_{\text {elast }}(Q)=A & {\left[4 j_{0}^{2}\left(Q r_{\text {eq }} / 2\right)\right.} \\
& \left.+4 / 3 j_{2}^{2}\left(Q r_{\text {eq }} / 2\right)\right] S_{\text {diff }}(Q, 0) .
\end{aligned}
$$

Here, a normal deuterium composition is assumed (i.e., the equilibrium para- $\mathrm{D}_{2}$ concentration of $1 / 3$, close to the value found for the solid runs, is assumed to be constant during the experiment), $A$ is a scaling constant, and $S_{\text {diff }}(Q, 0)$ is given by the mode-coupling equations (14). The value for the self-diffusion coefficient $D_{T}=0.3(1)$ $\AA^{2} \mathrm{ps}^{-1}$ is found from a fit which is in good agreement with the value of $0.28 \AA^{2} \mathrm{ps}^{-1}$, obtained from the width and amplitude of the incoherent part of the quasielastic line at larger wave vectors.

The contributions from the diffusive translational motions in the liquid phase are clearly apparent in the
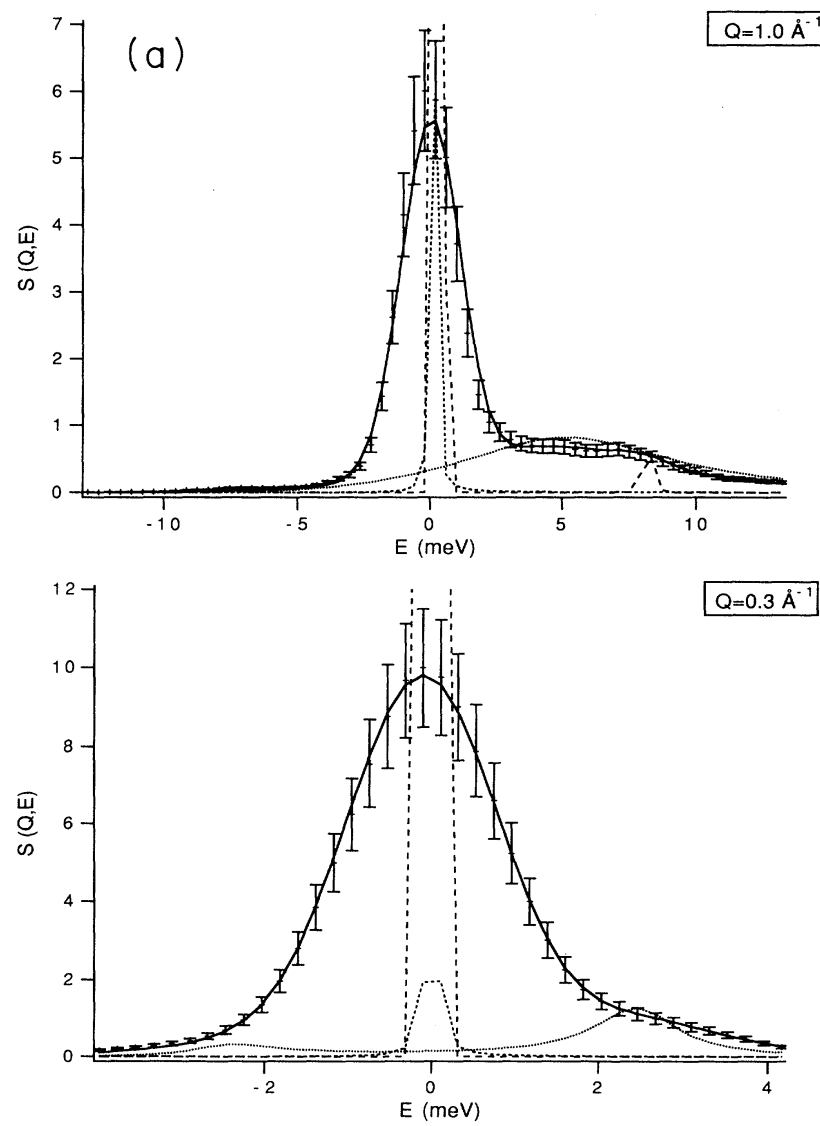

quantity $Z(E)$ as depicted in Fig. 3(a). It has a maximum at about $2.0 \mathrm{meV}$ and $Z(0)=0.065 \mathrm{meV}^{-1}$ from where a value of the diffusion coefficient may also be obtained. This contribution from translational diffusion closely resembles that one for classical liquids and several relevant parameters characterizing this motion can be derived from the analysis of this curve. In particular, the mean-intermolecular vibrational energy, $E_{0}$, may be estimated from a fit of the itinerant-oscillator model $^{27}$ to the measured data on function $Z(E)$. The fit yields $E_{0}=2.1 \mathrm{meV}$ corresponding to a frequency of $0.508 \times 10^{12} \mathrm{~Hz}$.

The heat capacity of the liquid has also been evaluated $^{28}$ and compared with the experimental data $^{29,30}$ as it is shown in Fig. 3(c). The neutron data are consistently above the experimental values as it should be expected since the heat capacities are evaluated in the harmonic approximation.

The low-energy collective dynamics can be reasonably well represented by the model scattering laws described above. However, systematic discrepancies appear on the neutron energy-loss side of the spectra corresponding to $Q$ values around the maximum of the structure function $S(Q)$. As a matter of fact, a hump located at about
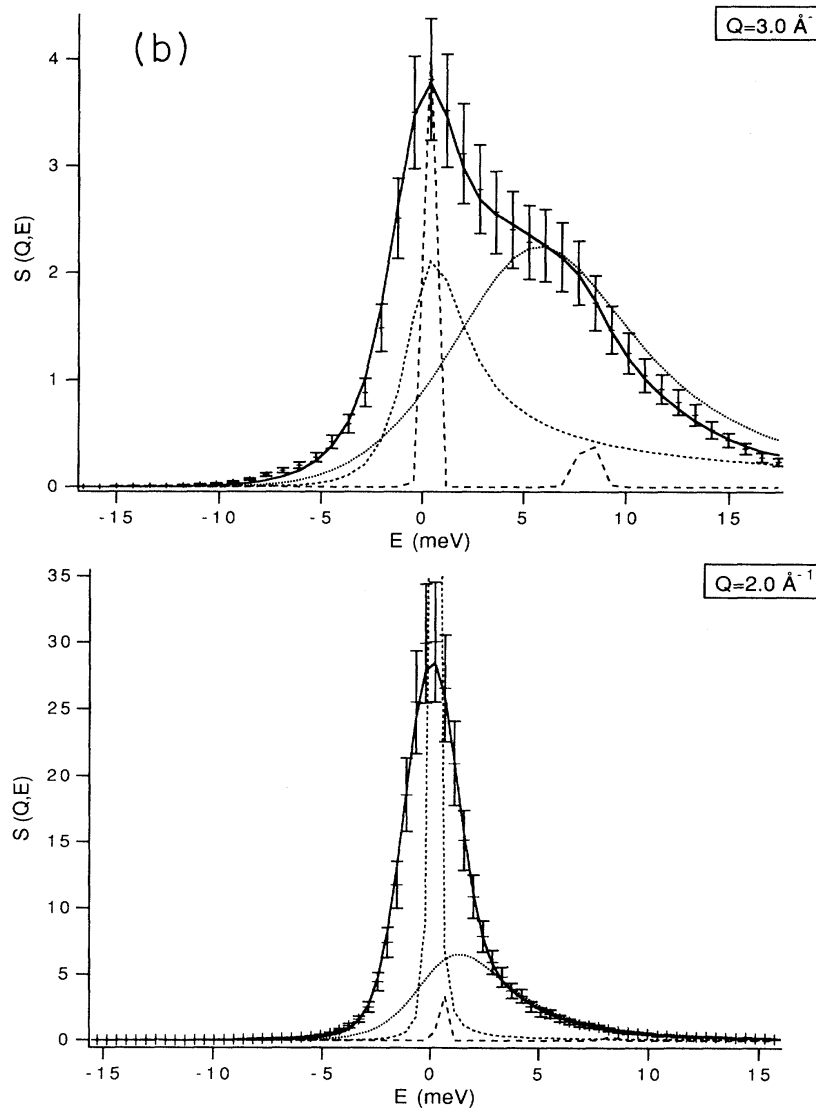

FIG. 5. Comparison of fitted and observed TAS spectra for liquid deuterium. The experimental points are shown with their respective error bars. The fitted model is shown by the solid line. The excitation spectrum which corresponds to the DHO is shown by the dotted line. The rotational contribution can be seen as a long-dash line which shows peaks at energy transfers of zero and $7.5 \mathrm{meV}$. The quasielastic contribution representing diffusive and low-energy coherent effects can also be seen as a Lorentzian (small dashes). 


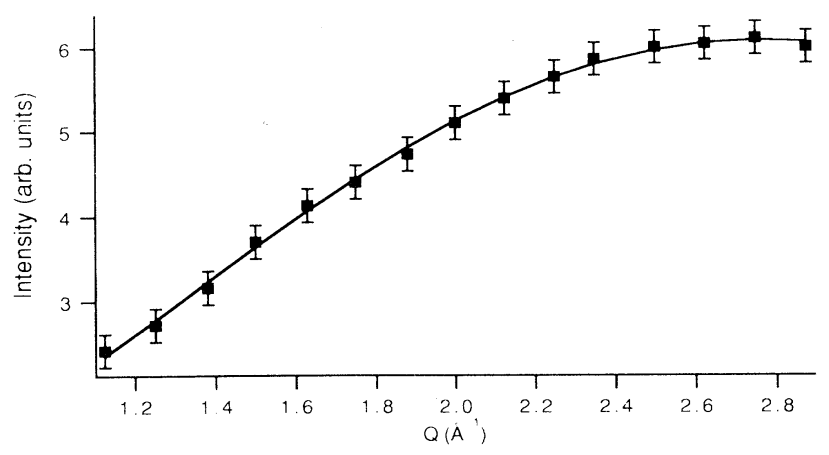

FIG. 6. Wave-vector dependence of the intensity for the $J=1 \rightarrow 0$ rotational peak in the solid at $T=10 \mathrm{~K}$. The solid line is a fit using the model given by Eq. (10).

$2.4 \mathrm{meV}$ can be seen in the spectrum corresponding to $Q=1.6 \AA^{-1}$ (Fig. 1) which does not show any measurable wave-vector dependence. Such an energy is too low to correspond to any of the lowest-lying phonon excitations which are located at about $4.8 \mathrm{meV}$, and it is obviously difficult to ascribe it to any significant form of collective effects. On the other hand, the energy-loss sides were in all cases well approximated by the model scattering functions.

The wave-vector dependence of the linewidth and amplitude of the coherent component of the quasielastic response are shown in Figs. 9(b) and 9(d), respectively. The most noticeable feature that can be seen is the substantial narrowing at a wave vector $Q_{p}$ that corresponds to the maximum of the static structure factor $S(Q)$ (i.e., de Gennes narrowing), and the approximate coincidence of the extrema of this function with those of the excitation curve discussed in the next section.

\section{B. Collective dynamics}

From inspection of the liquid phase spectra shown in Fig. 4 and measured using an incident energy of 34.9

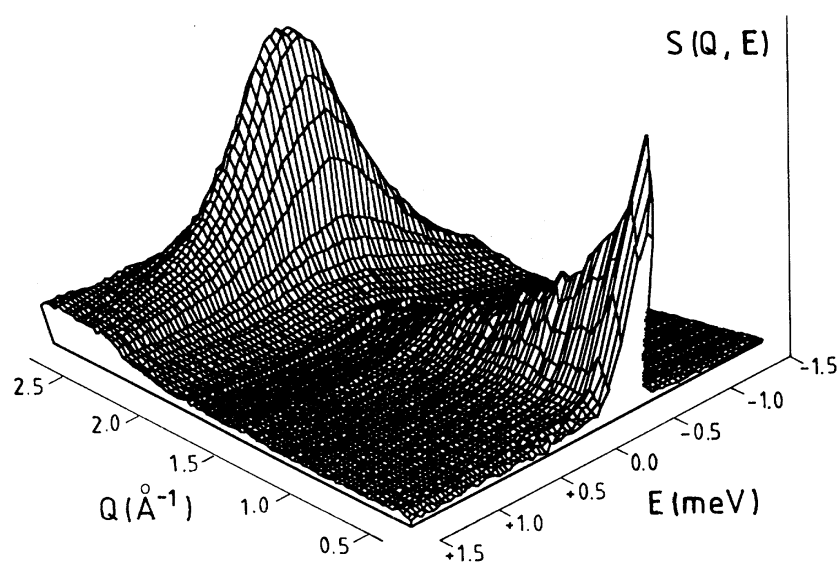

FIG. 7. $S(\Theta, E)$ surface for liquid deuterium at $T=20.14$ $\mathrm{K}$ illustrating the low-energy inelastic response.
meV, well above that characteristic of sound-mode excitations, it can be observed that

(i) A clear, though broadened, inelastic contribution is apparent on the neutron energy-loss side of the spectra for all the momentum-transfer values covered by the present experiment.

(ii) Such a finite-frequency feature which also contains superimposed to it the $J=0 \rightarrow 1$ rotational transition, shows dispersion to higher energies up to wave vectors of about $1.0 \AA^{-1}$. From $Q=1.0 \AA^{-1}$ to $Q=2.1 \AA^{-1}$, a shift of the peak position to lower energies is observed. From the latter value of $Q$ to the maximum value of momentum transfer achieved in this work $\left(Q=3.8 \AA^{-1}\right)$ a progression toward higher energies can be noticed.

(iii) The observed wave-vector dependence, therefore, cannot be attributed to recoil effects since these would evidence as a parabolic dependence on $Q$.

The wave-vector dependence of the renormalized excitation frequencies, $\Omega_{Q}$, as well as the damping factors, $\Gamma_{Q}$, are shown in Fig. 10. The approach to the hydrodynamic limit which is shown as a dashed line in the graph can be accounted for in terms of

$$
\frac{\hbar \Omega_{Q}}{k_{B}}=\frac{\hbar}{k_{B}} v_{T} Q+B_{d} Q^{2},
$$

where $B_{d}=47 \mathrm{~K} \AA^{2}$ is a measure of the departure from hydrodynamic behavior and $v_{T}=1090 \mathrm{~ms}^{-1}$ is the isothermal sound velocity. The phase velocity of the excitation
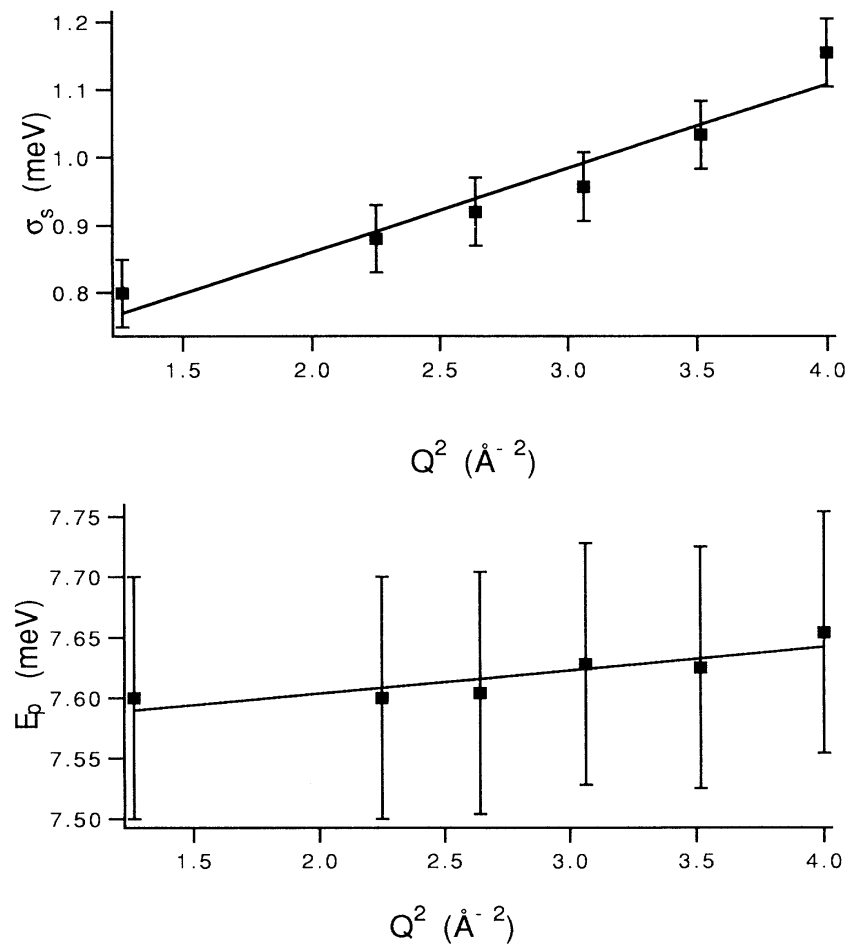

FIG. 8. Wave-vector dependence of the parameters characterizing the rotational peak in the liquid at $T=20.14 \mathrm{~K}$. The upper part shows the $Q$ dependence of the intrinsic width and the lower frame the transition energy. 
at low wave vectors is shown in Fig. 11. A dramatic amount of positive dispersion (i.e., sound velocities well above the hydrodynamic limit) is clearly seen up to wave vectors of about $1.0 \AA^{-1}$.

The measured velocity of sound thus correlates well with previously reported values for the adiabatic sound velocity measured by means of an ultrasonic technique ${ }^{31}$ of $v_{0}=1040 \mathrm{~ms}^{-1}$.

Near the minimum, the dispersion curve is well approximated by a parabolic function of type

$$
\hbar \Omega_{Q}=\hbar^{2}\left(Q-Q_{R}\right)^{2} / 2 M_{\mathrm{eff}}+\Delta
$$

in close analogy with the dispersion in the roton minimum of liquid ${ }^{4} \mathrm{He}$. The values characterizing the position of the minimum $Q_{R}$, the effective mass $M_{\text {eff }}$, and the energy gap $\Delta$ were found to be $2.08 \AA^{-1}, 0.072$ times the mass of a deuterium molecule, and $42.8 \mathrm{~K}$, respectively.

Beyond the roton minimum, some intensity is transferred from the one-phonon response to the multiphonon contribution, a fact that complicates the detailed analysis of this region if approximations more refined than the Gaussian are required. ${ }^{14}$ In particular, the mixing of multiphonon and recoil effects leads to difficulties in the accurate estimation of the $\Omega_{Q}$ frequencies and $\Gamma_{Q}$ damping factors for wave vectors above $2.5 \AA^{-1}$. The fact that the maxima of the broad multiexcitation contribution is located at $10.3 \mathrm{meV}$ and the relatively low temperature at which the experiment was performed, made this contribution rather small even at larger wave vectors. As a consequence, the spectral line shapes were well reproduced using the simplified approximation followed in this work.

The results on $S(Q, E)$ may be exploited to derive some information on the static structure function $S(Q)$ via the sum rule (zero moment)

$$
\int_{-\infty}^{+\infty} S(Q, E) d E=S(Q) .
$$

Integrating the fitted functions yields the curve in Fig. 12. It can be compared to theoretical results derived within the correlated density matrix theory ${ }^{32}$ on the intermolecular static structure function $S_{\mathrm{CM}}(Q)$. Adopting the approximation of uncorrelated rotational and translational motions, the total static structure function is given by ${ }^{33}$

$$
S(Q)=\frac{1}{2}+f_{1}(Q)+f_{2}(Q)\left[S_{\mathrm{CM}}(Q)-1\right],
$$

with the molecular form factors

$$
\begin{aligned}
& f_{1}(Q)=(1 / 2) j_{0}\left(Q r_{\mathrm{eq}}\right) \\
& f_{2}(Q)=\left[j_{0}\left(Q r_{\mathrm{eq}} / 2\right)\right]^{2}
\end{aligned}
$$

Figure 12, upper frame, gives a comparison of the theoretical and experimental center-of-mass function $S_{\mathrm{CM}}(Q)$.

We may also compare the results on the excitation energy $\hbar \Omega_{Q}$, the phase velocity $c(Q)$ (Fig. 11), the amount of dispersion, the strength factor $H(Q)$, and the vari-
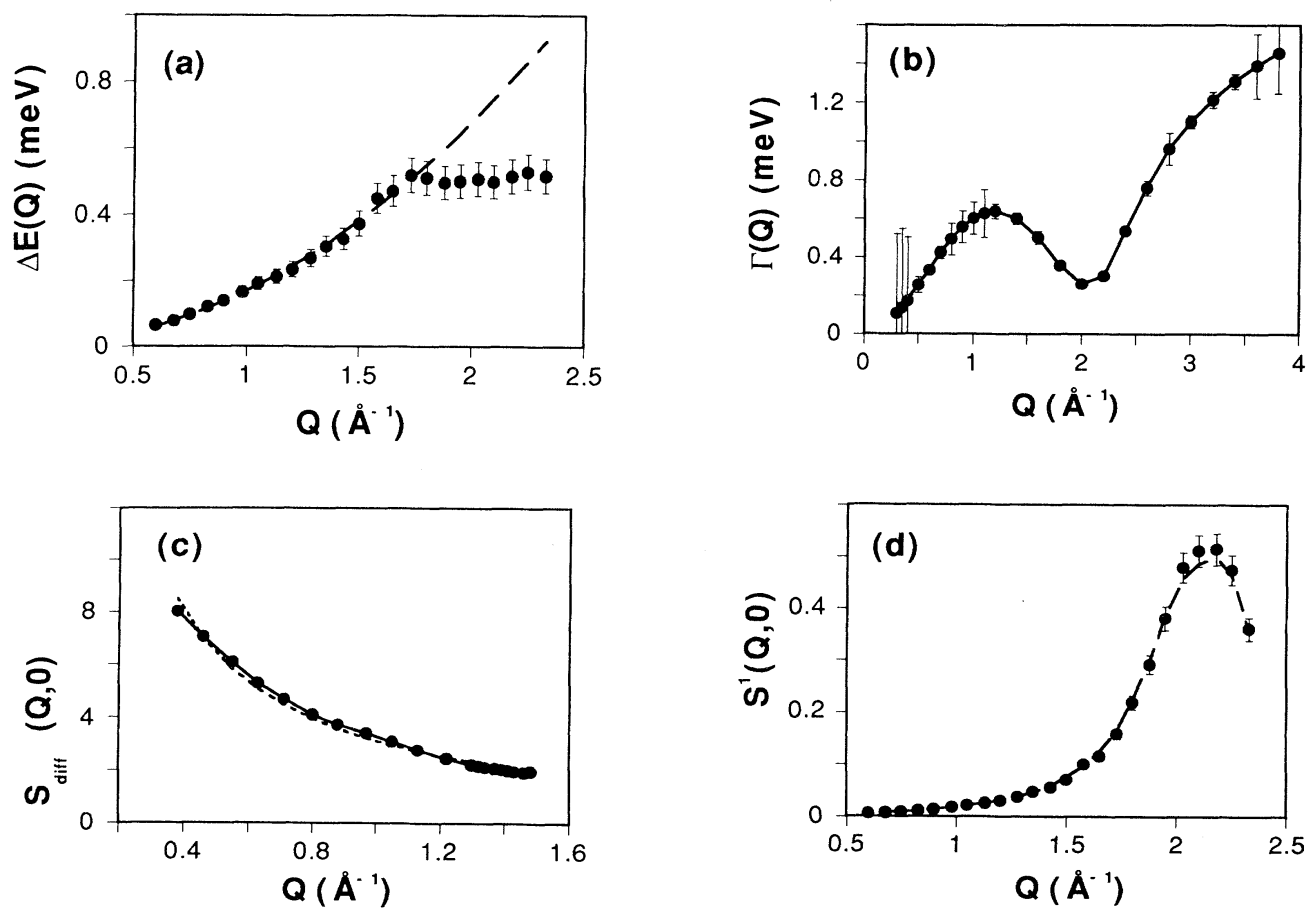

FIG. 9. $Q$ dependence of the parameters which characterize the quasielastic scattering in liquid deuterium. (a) Incoherent linewidth and its calculated dependence using Eq. (12), (b) linewidth of the coherent quasielastic structure factor [see Eq. (4)]. The values above $Q=2.4 \AA^{-1}$ correspond to estimates from the analysis of the TAS spectra. (c) Elastic intensity at low momentum-transfer values [see Eq. (13)] and (d) total amplitude for elastic scattering of the low-energy spectra [see Eq. (4)]. 
ous sum rules with theoretical results on these quantities derived within the correlated density matrix theory. ${ }^{32,34}$ At present, this approach is realized on the level of the single-mode approximation, Eq. (8), without damping,

$$
\begin{aligned}
& D\left(Q, E^{2}\right)=\delta\left\{E^{2}-\left[E^{\mathrm{th}}(Q)\right]^{2}\right\} \\
& H(Q)=S_{\mathrm{CM}}^{\mathrm{th}}(Q) \tanh \left(\frac{\beta}{2} E^{\mathrm{th}}(Q)\right) .
\end{aligned}
$$

The calculation has been done at a temperature $T=20 \mathrm{~K}$ and a (molecular) number density $\varrho=0.027 \AA^{-3}$. The latter should be comparable to the experimental density corresponding to the experimental pressure $p=2$ bars. Our results on the excitation energy $E^{\text {th }}(Q)$ are shown
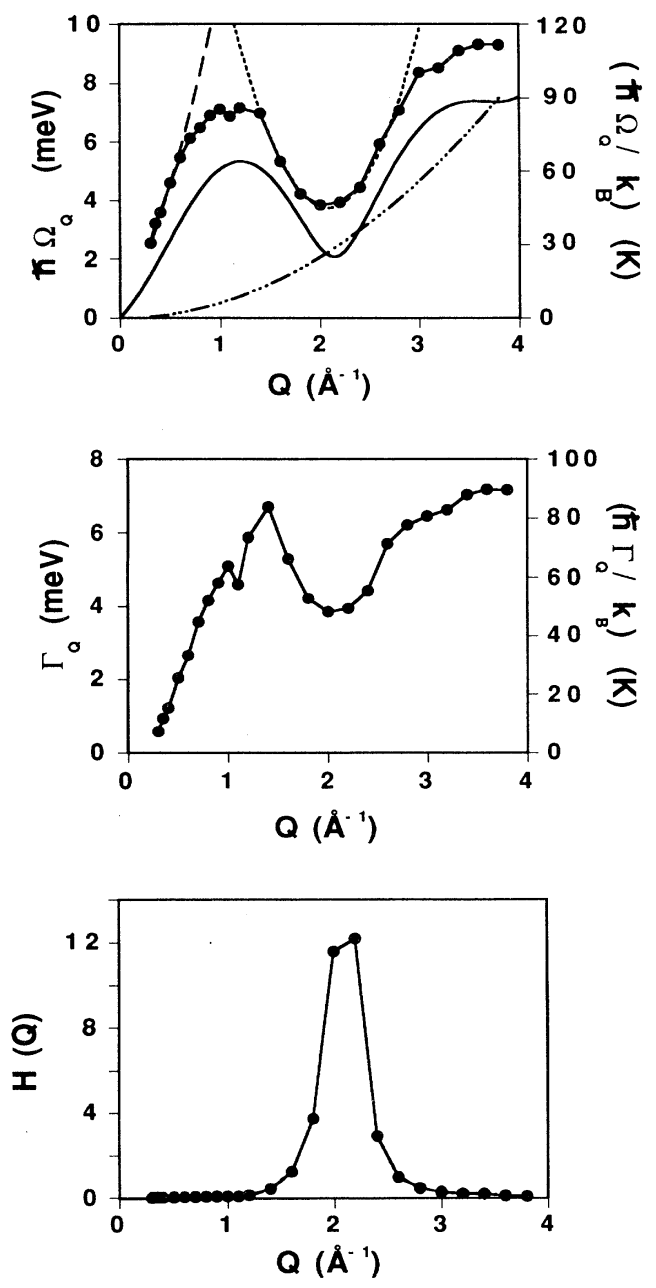

FIG. 10. The upper part shows the excitation energies (solid line and full circles) and the recoil curve for a particle with a mass equal to that of the deuterium molecule (dashdots) The results from the density-matrix calculation is given by the solid line. The curves above the experimental results are the fits to the roton [Eq. (17)] and phonon [Eq. (16)] parts of the dispersion curve. The middle frame of the figure shows the damping factors, and the lower frame shows the $H(Q)$ one-phonon structure factor.
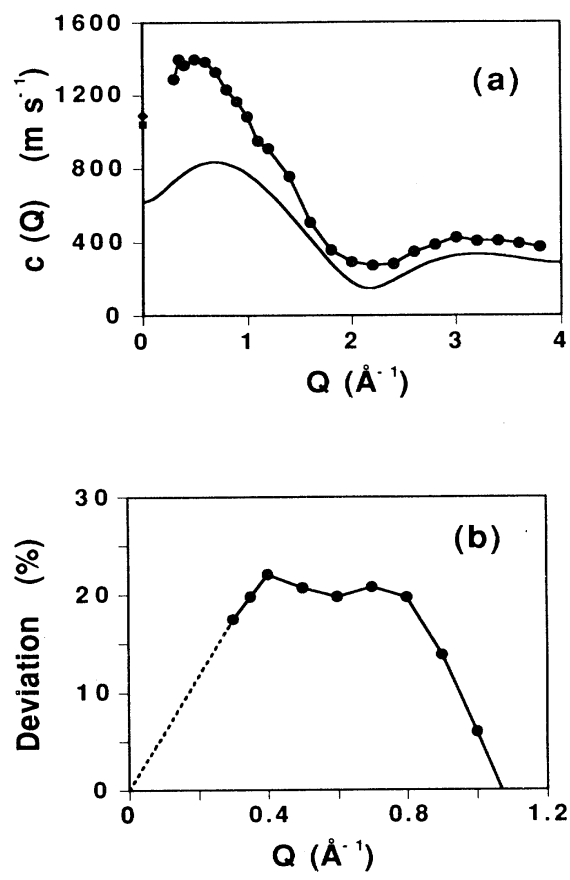

FIG. 11. (a) Excitation phase velocities (solid line and full circles) derived from the TAS results. The filled square shows the value of the adiabatic velocity. The curve corresponding to the calculation is shown as the solid line below. (b) Deviation from hydrodynamic sound (positive dispersion) expressed in percent.

in Fig. 10 while the phase velocity $c(Q)=E^{\text {th }}(Q) / \hbar Q$ is compared to the experimental values in Fig. 11. The comparison of the experimental and theoretical excitation energies, $\hbar \Omega_{Q}$ and $E^{\text {th }}(Q)$, respectively, shows fair agreement in the general shape of the curves, in particular in the locations of the maximum ("maxon region") and minimum ("roton region") in $Q$ space, whereas systematic differences clearly occur in the energy values, especially in the phonon and maxon regions. To some extent, this is unexpected, as it is well known that the neglect of backflow ${ }^{35}$ fails to bring down the Feynman rotons to the experimental values but has little effect on the phonons in liquid ${ }^{4} \mathrm{He}$. However, once this discrepancy in the overall scaling of the two curves is accepted, the comparison shown in Fig. 11 for the phase velocities of the excitations evidences the fact that the large amount of positive dispersion shown by the experimental data is reproduced in the calculation although the calculation seems to deviate from the hydrodynamic limit a great deal more than an extrapolation of the experimental behavior.

\section{SUMMARY AND DISCUSSION}

\section{A. Low-energy dynamics}

The quasielastic response of liquid and solid deuterium has been studied by means of TOF neutron scattering, and the spectra have been analyzed on a quantitative 
basis. Although most of the spectral features have been taken into account, small but significant deviations from the model-scattering law still persist. In particular, the low, finite-frequency response observed in the liquid by TOF at momentum-transfer values around $Q_{p}$, the $Q$ value for the maximum in $S(Q)$ (see Fig. 1) remains to be assigned to a collective dynamical process.

From the study of the inelastic rotational peak in the solid and liquid phases, it has been shown that small but noticeable dependences with the wave vector exist and, therefore, can be exploited for detailed studies on the ortho- and para-concentration dependences of the peak parameters. In this respect, TOF neutron spectroscopy could provide complementary information to that derived from NMR on solid mixtures of ortho- and para- $\mathrm{H}_{2}$ and $\mathrm{D}_{2}$, on these systems which are considered to be clear examples of the orientational glassy state. ${ }^{36}$ Additional studies in the solid phases of both $\mathrm{H}_{2}$ and $\mathrm{D}_{2}$ using higher energy resolution could provide valuable information regarding the magnitude of such effects as rotationaltranslation coupling.

The coherent quasielastic response of this liquid can only be understood in semiquantitative terms since a model for collective reorientational motion at this length scale is still lacking.

The quantum effects on this system have already manifested as the rather large values of the amplitude terms entering the Debye-Waller factors in the solid phase. Although the rotational motion is a well-known quantum characteristic in these system, the diffusional behavior in the liquid phase can be well understood in classical terms.

\section{B. Collective dynamics}

The collective character of the excitations observed by means of TAS spectroscopy is manifested by the strong wave-vector dependence of the inelastic intensities illustrated in Fig. 4. This dependence cannot be accounted for solely in terms of single-molecule inelastic contributions to the scattering cross section, although, from a cursory glance, the origin of the observed intensities at certain values of the momentum transfer ( $Q$ values about $1.0 \AA^{-1}$ ) could be tentatively assigned to rotational transitions. Such an attempt would not stand a comparison with spectra outside those particular values of $Q$ and would result in ratios of the intensities for the $\Delta J=0$ and $\Delta J=1$ rotational transitions well away from the theoretical values predicted from the formulas by Elliot and Hartmann. ${ }^{1}$ Further support for the collective nature of the observed response comes from simple considerations regarding the frequency moments of the experimental spectra shown in Fig. 12. While no $Q$ dependence is found which can be identified as corresponding to ro-
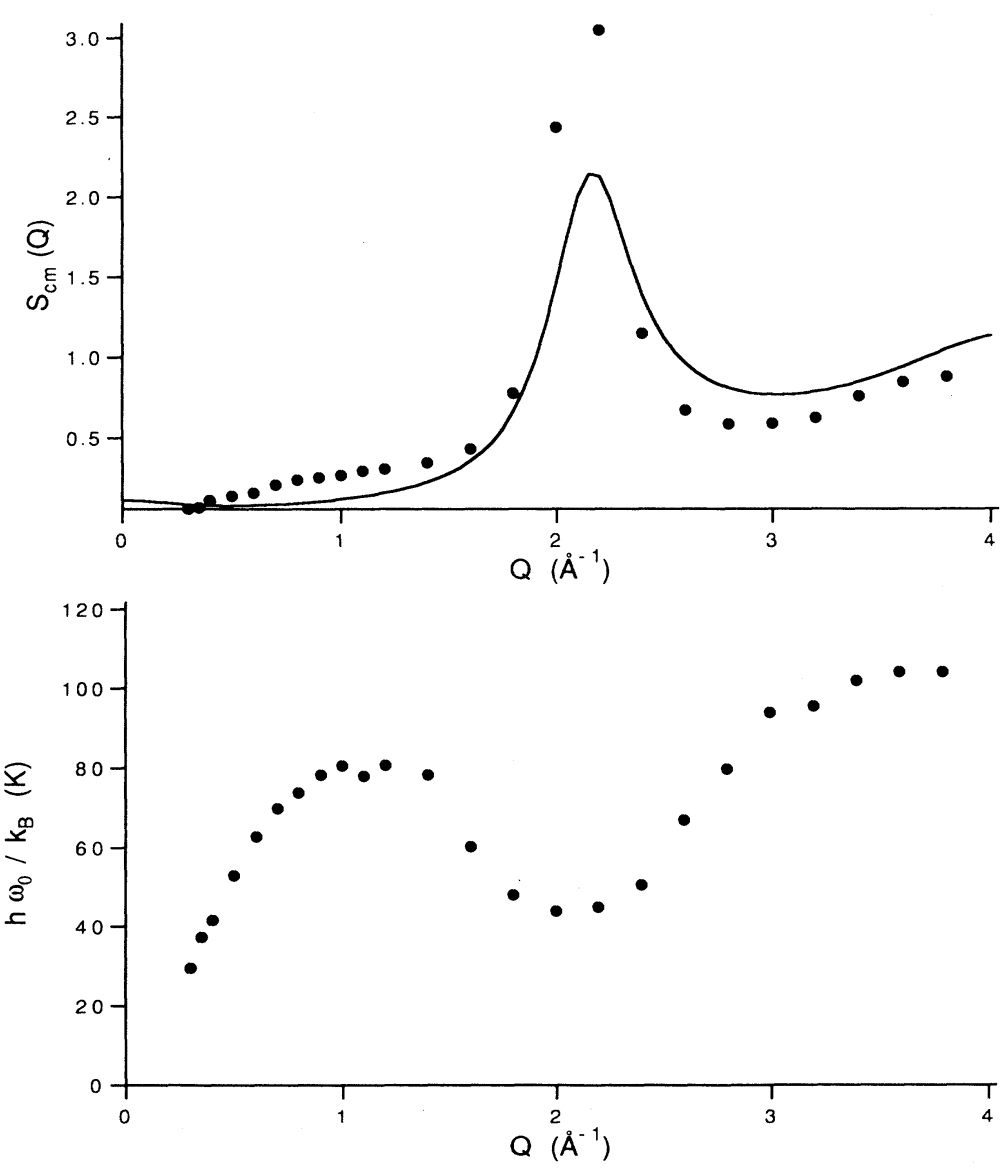

FIG. 12. The first frequency moments of the $S(Q, E)$ dynamic structure factors. The upper graph shows the estimate of the $S_{\mathrm{CM}}(\mathrm{Q})$, static structure factor for the center of mass from the calculation (solid line) and from the experimental results (full circles). The lower frame displays the normalized second frequency moment (i.e., the ratio of second to zeroth moments). 
tational transitions, the structure factor and the "dispersion relation" showing a characteristic minimum at $Q$ values near the maximum in $S(Q)$ are recovered.

From the values of the renormalized excitation frequency $\Omega_{Q}$ and damping factor $\Gamma_{Q}$ (Ref. 37) of the collective density excitation two different regions within the explored momentum-transfer range can be distinguished: a small damping $\left(\Gamma_{Q}^{2}<\hbar^{2} \Omega_{Q}^{2} / 2\right)$ region which extends up to $Q$ values of $1.1 \AA^{-1}$ where well-defined inelastic side peaks are clearly visible in the TAS spectra and an intermediate region where $\hbar^{2} \Omega_{Q}^{2} / 2<\Gamma_{Q}^{2}<\hbar^{2} \Omega_{Q}^{2}$, corresponding to spectra where the inelastic contribution is apparent, although well-resolved peaks are not present. Such a region covers $Q$ values from $Q=1.1 \AA^{-1}$ to 3.8 $\AA^{-1}$, although in the present case it becomes difficult to distinguish if such inequality still holds near the minimum in the dispersion curve. The finite-energy feature clearly apparent in the spectra below this value of the momentum transfer becomes difficult to distinguish about 2 $\AA^{-1}$ due to a strong reduction in the bare frequency. This reduction is normally observed even in classical liquids when the maximum of the structure factor is approached (see Refs. 38 and 39 for two representative examples).

The dispersion behavior shown in Fig. 10 can be correlated with those reported for solid $D_{2}$ (Ref. 5) at zero and 275 bars of applied pressure. As a matter of fact, the maximum excitation frequency correlates well with those measured in the solid phase at the Brillouin zone boundary. The density of vibrational states, $Z(E)$, has also been calculated from the measured TAS spectra following the approximation given in Eq. (7), and the resulting curve is shown in Fig. 13. From comparison between the $Z(E)$ displayed in Fig. 3(b) for liquid deuterium and the curve shown in Fig. 13 corresponding to the density of states derived from the fitted functions to the TAS spectra (i.e., without effects due to instrumental broadening) it becomes apparent that a large number of excitations appear between the low-frequency region, which is dominated by low-energy coherent excitations which also appear in the TOF data and the $J=1 \rightarrow 0$ rotational peak.

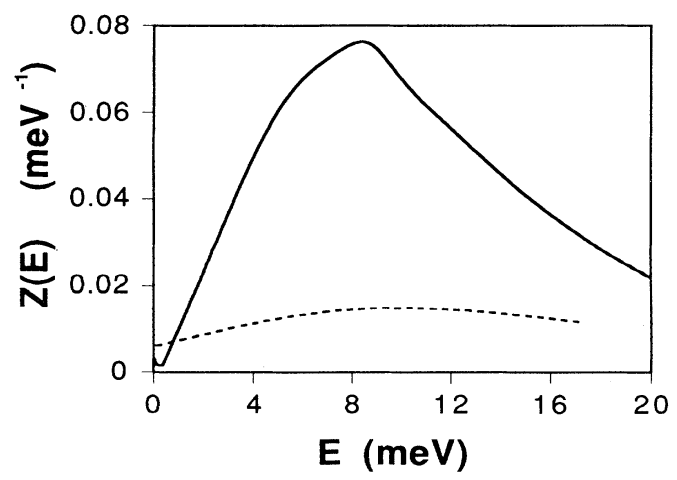

FIG. 13. $\quad Z(E)$ vibrational density of states derived from the TAS measurements. The solid line shows the result of the calculation of $\mathrm{Z}(\mathrm{E})$ from the fitted model scattering law. It should be compared with the density of states shown in Fig. 3 (b). The dashed line below represents the multiexcitation contribution which has been magnified ten times.
Such excitations correspond to vibrational states weakly populated at $T=20.14 \mathrm{~K}$ (a calculation of the occupation number for frequencies corresponding to the maximum of the dispersion curve gives a population factor of about $1 \%$ ), so that their observation only becomes possible if the energy of the impinging neutrons is higher than about $7.8 \mathrm{meV}$.

The dispersion curve measured in the present work can also be compared with the one reported for liquid ${ }^{4} \mathrm{He}$ in the normal liquid phase at $T=3.1 \mathrm{~K} .{ }^{40}$ The reported maximum excitation frequency occurs at about $1.2 \AA^{-1}$ and a minimum is seen at about $2 \AA^{-1}$, which are rather close to the results presented in this work. The corresponding excitation frequencies, once the different definitions of $\Omega_{Q}$ are taken into account, are about 25.4 and $21 \mathrm{~K}$, respectively, which are, as expected, far lower than the ones for liquid $\mathrm{D}_{2}$. However, the relative contribution of the damping terms seems to be somewhat more important for normal liquid ${ }^{4} \mathrm{He}$ than for liquid $\mathrm{D}_{2}$. Such a fact can be understood from consideration of the relative values of the reduced temperature $T^{*}=k_{B} T / \epsilon_{0}$, where $\epsilon_{0}$ is the parameter characterizing the depth of the intermolecular potential, and the microscopic Grüneisen parameter $\gamma_{G}$ which accounts for anharmonic effects. Since the damping effects are known to depend strongly on both the thermal population and anharmonicity, it is clear that the damping effects become stronger for larger values of $T^{*}$ and $\gamma_{G}$. The reduced temperatures for both experiments are, respectively, 0.31 for ${ }^{4} \mathrm{He}$ at $T=3.1 \mathrm{~K}$ and 0.55 for liquid $\mathrm{D}_{2}$ where the standard value for the potential parameter of $\epsilon_{0} / k_{B}=36.7 \mathrm{~K}$ has been used. On the other hand, the Grüneisen parameter for ${ }^{4} \mathrm{He}$ at the reported temperature has been estimated to be equal to 3.1 , whereas an estimation using data for solid $\mathrm{D}_{2}$ (Ref. 4) gives a value of $\gamma_{G}=1.63$. It seems therefore that although the thermal population parameter would lead to stronger damping effects in liquid $\mathrm{D}_{2}$ than in normal liquid ${ }^{4} \mathrm{He}$, the anharmonicity contribution can effectively counterbalance this effect. These reflections are also in line with the comments made by Pedersen and Carnerio, ${ }^{40}$ about the intercomparison between normal ${ }^{4} \mathrm{He}$ and liquid $\mathrm{Ar}$ and $\mathrm{Rb}$ as to the observability of collective excitations. In fact, liquid $\mathrm{D}_{2}$ seems to support collective excitations under conditions of smaller thermal population and smaller anharmonicity than liquid $\mathrm{Rb}$.

A quantitative comparison between the correlateddensity-matrix theoretical approach and experiment is difficult due to the different meaning of the excitation frequency in both cases. Since the model-scattering law employed for the analysis of the inelastic response is based upon a damped harmonic oscillator response function, it seems natural to assign a physical meaning to the renormalized excitation frequency $\Omega_{Q} \cdot{ }^{37}$ On the other hand, the calculations have been performed in the limit of no damping and an intermolecular potential function of a pure Lennard-Jones type has been used, which ignores electrostatic (quadrupolar) interactions generated by the presence of rotationally excited para- $\mathrm{D}_{2}$ molecules. But these very interactions are responsible for the broadening of the low-energy spectra in mixtures of ortho and para components. The presence of substantial amounts 
of para- $\mathrm{D}_{2}$ is known to increase the number density of the liquid $^{41}$ and such a fact can partially explain the difference between the calculated and measured curves. Additionally, since the static structure function $S(Q)$ has been calculated within an integral equation approach based on a restricted trial ansatz for the density matrix and by adopting a number of approximations, such as the HNC/0 approximation and the separability assumption (see Appendix), some systematic discrepancies between theory and experiment are expected. It is now known, e.g., that the closure relationships derived from integral equation approaches, are unable to reproduce the measured structure factors with the statistical accuracy nowa-days available in diffraction experiments. ${ }^{42}$

Although a more refined version of the theory is definitely needed to enable a quantitative comparison, some relevant features are clearly apparent in results from both theory and experiment. In particular, the large amount of positive dispersion at low-momentum-transfer values is now substantiated in both cases, and the percentage deviation from hydrodynamic behavior is now confirmed to be far larger than the one found for liquid ${ }^{4} \mathrm{He}$ in the superfluid phase, where the most recent estimate is about $4 \% .{ }^{43}$

\section{ACKNOWLEDGMENTS}

This work has been supported in part by DGICYT Grant No. PB89-0037-C03 and by the Deutsche Forschungsgemeinschaft under Grant No. Ri 267/16-1. The help given by Dr. A.J. Dianoux of the Institut Laue Langevin during the TOF experiments is warmly acknowledged. Discussions with Dr. B. Fåk from the Centre d'Etudes Nucleaires, Grenoble, is also acknowledged.

\section{APPENDIX: THE CORRELATED-DENSITY-MATRIX APPROACH}

Within the framework of this approach, the elementary excitation energy $E^{\text {th }}(Q)$ and the structure function $S_{\mathrm{CM}}^{\mathrm{th}}(Q)$ are optimally determined by solving two coupled Euler-Lagrange equations with the intermolecular potential as microscopic input. Both quantities are, at present, described within the Feynman approximation in conjunction with the HNC/0 approximation. Thus, backflow is ignored. The equations have the form of a generalized Feynman equation

$E^{\text {th }}(Q) \tanh \left(\frac{\beta}{2} E^{\text {th }}(Q)\right)=E_{\mathrm{kin}}(Q) / S_{\mathrm{CM}}^{\mathrm{th}}(Q)$,
$E_{\text {kin }}(Q)$ being the kinetic energy of a deuterium molecule, and of a paired-phonon condition

$$
\frac{1}{2} E_{\mathrm{kin}}(Q)\left[1-S_{\mathrm{CM}}^{\mathrm{th}}(Q)\right]=\dot{S}_{\mathrm{CM}}^{\mathrm{th}}(Q),
$$

with a generalized structure function $\dot{S}_{\mathrm{CM}}^{\text {th }}(Q)$ defined explicitly in Ref. 32. The solutions yield the optimal excitation energy $E^{\text {th }}(Q)$ and optimal structure function $S_{\mathrm{CM}}^{\text {th }}(Q)$ (in HNC/0 approximation) for a correlated $N$ particle trial density matrix of Jastrow form

$$
\begin{aligned}
W\left(\mathbf{R}, \mathbf{R}^{\prime}\right)=\exp \{ & \sum_{i<j}^{N} \frac{1}{2} u\left(\left|\mathbf{r}_{i}-\mathbf{r}_{j}\right|\right)+\sum_{i, j}^{N} \gamma\left(\left|\mathbf{r}_{i}-\mathbf{r}_{j}^{\prime}\right|\right) \\
& \left.+\sum_{i<j}^{N} \frac{1}{2} u\left(\left|\mathbf{r}_{i}^{\prime}-\mathbf{r}_{j}^{\prime}\right|\right)\right\} / I_{N},
\end{aligned}
$$

where $\mathbf{R}=\left(\mathbf{r}_{1}, \ldots, \mathbf{r}_{N}\right)$ denotes the positions of $N \mathrm{D}_{2}$ molecules that are distributed in a large volume with a homogeneous number density $\varrho$. For simplicity we assume that the molecules interact with each other, in some angle-averaged sense, via a pair potential of LennardJones form

$$
v(r)=4 \epsilon_{\mathrm{LJ}}\left[\left(\frac{\sigma_{\mathrm{LJ}}}{r}\right)^{12}-\left(\frac{\sigma_{\mathrm{LJ}}}{r}\right)^{6}\right],
$$

with parameters $\epsilon_{\mathrm{LJ}}=35.24 \mathrm{~K}$ and $\sigma_{\mathrm{LJ}}=2.958 \AA$ advocated in Ref. 44 , and we also ignore the rotational degrees of freedom for the present purpose.

The normalization integral $I_{N}$ appearing in Eq. (25) ensures that we have the property $\operatorname{Tr}\{W\}=$ $\int d \mathbf{R} W(\mathbf{R}, \mathbf{R})=1$, and the input functions $u(r)$ and $\gamma(r)$ are chosen to minimize the Helmholtz-free energy

$$
F[W]=\operatorname{Tr}\left\{W H+\beta^{-1} W \ln W\right\}
$$

associated with the trial statistical operator $W$ at a given temperature $T=k_{B} \beta^{-1}$. This procedure is motivated by the Gibbs-Delbrück-Molière minimum principle, stating that quantity (27) establishes an upper bound for the exact free energy calculated from the equilibrium statistical operator $W_{0}=\exp \{-\beta(H-F)\}$ associated with the Hamiltonian $H$ of the system. As described in detail in Ref. 32, the optimization is performed within the separability approximation, leading to the Euler-Lagrange equations (23) and (24).

We have numerically solved these equations using the same iteration scheme that has proved successful in the liquid ${ }^{4} \mathrm{He}$ (Ref. 32) and $\mathrm{H}_{2}$ (Ref. 45) problems.

\footnotetext{
*Permanent address: Departamento de Física Aplicada C-IV, Facultad de Ciencias, Universidad Autónoma de Madrid, E-28049, Madrid, Spain.

${ }^{1}$ Theoretical and experimental studies concerning the dynamics of condensed hydrogen and deuterium are reported in R.J. Elliot and W.M. Hartman, Proc. Phys. Soc. London 90, 671 (1967); P.A. Egelstaff, B.C. Haywood, and F.J.
}

Webb, Proc. Phys. Soc. London 90, 681 (1967). Note that to compare the values for the line widths given in this work with those reported in the above referred papers, a conversion factor of 2.538 has to be used in order to convert the Gaussian full widths to frequency moments. A number of other studies have appeared, for instance, see W. Schott, Z. Phys. 231, 243 (1970) and references therein. Studies of 
hydrogen dissolved on an inert matrix or in a gas phase by neutron scattering are reported by V.F. Sears, Proc. Phys. Soc. London 86, 965 (1965); S.H. Chen, T.A. Postol, and K. Skold, Phys. Rev. A 16, 2112 (1977); J.A. Young and J.U. Koppel, Phys. Rev 135, 603 (1973). A set of models for the scattering of hydrogen can also be found in G. Sarma, Inelastic Scattering of Neutrons in Solids and Liquids (I.A.E.A., Vienna, 1961), p. 397; V.F. Sears, Can. J. Phys. 44, 1279 (1966).

${ }^{2}$ P. Verkerk, U. Bafile, B. Farago, and F. Mezei, Physica B 168, 1 (1991).

${ }^{3}$ W. Langel, D.L. Price, R.O. Simmons, and P.E. Sokol, Phys. Rev. B 38, 11275 (1988).

${ }^{4}$ M. Nielsen, Phys. Rev. B 7, 1626 (1973); A. Bickerman, H. Spitzer, H. Stiller, H. Meyer, R.E. Lechner, and F. Volino, Z. Phys. 31, 345 (1978).

${ }^{5}$ K. Carneiro, M. Nielsen, and J.P. McTague, Phys. Rev. Lett. 30, 481 (1973).

${ }^{6}$ F.J. Bermejo, J.L. Martinez, D. Martin, F.J. Mompean, M. Garcia-Hernandez, and A. Chahid, Phys. Lett. A 158, 253 (1991).

${ }^{7}$ H.J. Maris, G.M. Seidel, and T.E. Huber, J. Low Temp. Phys. 51, 471 (1983). Supercooling of small droplets of hydrogen has been reported by H.J. Maris, G.M. Seidel, and F.I.B. Williams, Phys. Rev. B 36, 6799 (1987).

${ }^{8}$ P. Sindzingre, D.M. Ceperley, and M.L. Klein, Phys. Rev. Lett. 67, 1871 (1991).

${ }^{9}$ Institut Laue Langevin software. Program INX.

${ }^{10}$ M.W. Johnson, AERE Report No. 7682 (1974).

${ }^{11}$ Computer codes developed by F. Rieutord, Institut Laue Langevin (private communication).

${ }^{12} \mathrm{X}$. Torrelles (unpublished).

${ }^{13} \mathrm{~A}$ modified version of the RESCAL code is briefly described in F.J. Bermejo, F. Batallan, J.L. Martinez, M. GarciaHernandez, and E. Enciso, J. Phys: Condens. Matter 2, 6659 (1990).

${ }^{14}$ S. W. Lovesey, Theory of Neutron Scattering From Condensed Matter (Oxford University Press, Oxford, 1986).

${ }^{15}$ Since the DOS measured from the energy-gain side of the spectra due to the relatively low temperature of the measurements (i.e., the adiabatic sound velocity at $T=20 \mathrm{~K}$ corresponds to an energy of about $80 \mathrm{~K}$ ) does not show a clear presence of one-phonon excitations, we have taken the density of states of solid para- $\mathrm{H}_{2}$ displayed in Fig. 3 of Bickerman et al. (Ref. 4) for the multiphonon calculation. Although the result of such calculations should be taken as approximate, it can be taken as an estimate of the relative importance of the multiexcitations.

${ }^{16}$ W.I.F. David and J.C. Matthewman, J. Appl. Crystallogr. 18, 461 (1985).

${ }^{17}$ J.G. Powles and G. Rickayzen, Mol. Phys. 32, 301 (1972).

${ }^{18}$ U. Buchenau, Z. Phys. B 58, 181 (1985).

${ }^{19}$ K. Carneiro, Phys. Rev. A 14, 517 (1976).

${ }^{20}$ I.F. Silvera, Rev. Mod. Phys. 52, 393 (1980).

${ }^{21}$ J. van Kranendonk and G. Karl, Rev. Mod. Phys. 40, 531 (1968).

${ }^{22}$ H. Stein, H. Stiller, and R. Stockmeyer, J. Chem. Phys. 57, 1726 (1972); A. Bickerman, H. Spitzer, H. Stiller, and R. Scherm, Z. Phys. B 31, 339 (1978).

${ }^{23}$ K.N. Klump, O. Schnepp, and L.H. Nosanow, Phys. Rev. B 31, 339 (1978).
${ }^{24}$ I. Ishmaev, I.P. Sadicov, A.A. Chernyshov, S.L. Isakov, B.A. Vindryaevskii, G.V. Kobelev, V.A. Sukhoparov, and A.S. Telepnev, Zh. Eksp. Teor. Fiz. 94, 190 (1988) [Sov. Phys. JETP 68, 1403 (1988)].

${ }^{25}$ I.M. de Schepper and M.H. Ernst, Physica A 98, 189 (1979).

${ }^{26}$ P. Verkerk, J.H. Builtjes, and I.M. de Schepper, Phys. Rev. A 31, 1731 (1991).

${ }^{27}$ V.F. Sears, Proc. Phys. Soc. London 86, 953 (1965).

${ }^{28}$ The heat capacity was calculated using

$$
C(T)=6 R \int_{0}^{\omega_{m}} \frac{x^{2} \exp (-x)}{[\exp (-x)-1]^{2}} Z(\omega) d \omega
$$

where $\omega_{m}$ is the maximum frequency which was taken as 20 $\mathrm{meV}, R$ stands for the gas constant, and $x=\hbar \omega / k_{B} T$. The normalization to $6 R$ is appropriate for a molecular solid, and is also used for the liquid.

${ }^{29}$ E.C. Kerr, E.B. Rifkin, H.L. Johnston, and J.T. Clarke, J. Am. Chem. Soc. 73, 282 (1951).

${ }^{30}$ H.M. Roder, G.E. Childs, R.D. McCarty, and P.E. Angerhofer, Nat. Bur. Standards Technical Note No. 641 (1973).

${ }^{31}$ D. Gusewell, F. Schmeissner, and J. Schmidt, Cryogenics 10, 150 (1970). The value for the adiabatic sound velocity has been estimated from extrapolation to $T=20.14 \mathrm{~K}$ of data given in Table I.

${ }^{32}$ G. Senger, M.L. Ristig, K. Kürten, and C.E. Campbell, Phys. Rev. B 33, 7562 (1986).

${ }^{33}$ P.A. Egelstaff, D.I. Page, and J.G. Powles, Mol. Phys. 20, 881 (1971).

${ }^{34}$ G. Senger, M.L. Ristig, C.E. Campbell, and J.W. Clark, Ann. Phys. (N.Y.) 218, 160 (1992).

${ }^{35} \mathrm{P}$. Nozières and D. Pines, The Theory of Quantum Liquids, Volume II: Superfluid Bose Liquids (Addison-Wesley, Redwood, California, 1990).

${ }^{36}$ U.T. Hochli, K. Knorr, and A. Loidl, Adv. Phys. 39, 405 (1990).

${ }^{37} \mathrm{~B}$. Fåk and B. Dorner (unpublished). The strength of the scattering function for a collective excitation in a liquid can only be calculated from the $f$ sum rule in the no-damping limit. In such a case, the sum rule gives for the excitation amplitude $H(Q)=Q^{2} /\left(2 M \Omega_{Q}\right)$.

${ }^{38}$ J.R.D. Copley and J.M. Rowe, Phys. Rev. Lett. 32, 49 (1974).

${ }^{39}$ T. Bodensteiner, C. Morkel, and W. Glaeser, Phys. Rev. A 45, 5709 (1992).

${ }^{40}$ K.S. Pedersen and K. Carneiro, Phys. Rev. 22, 191 (1980).

${ }^{41}$ S.J. Talhouk, P.M. Harris, D. White, and R.A. Erickson, J. Chem. Phys. 48, 1273 (1968).

${ }^{42} \mathrm{~A}$ comparison of the predictive capability of RISM integral equations using $\mathrm{HNC}$ and PY closures is given in F.J. Bermejo, E. Enciso, J. Alonso, N. Garcia, and W.S. Howells, Mol. Phys. 64, 1169 (1988).

${ }^{43}$ W.G. Stirling, in 75th Jubilee Conference on Helium-4, edited by J.G.M. Armitage (World Scientific, Singapore, 1983), p. 109.

${ }^{44}$ P.C. Souers (unpublished).

${ }^{45}$ M.L. Ristig, G. Senger, and K.E. Kürten, in Recent Progress in Many-Body Theories, edited by A.J. Kallio et al. (Plenum, New York, 1988), Vol. 1, p. 197. 